\title{
Amplitude modulation of androgen signaling by c-MYC
}

\author{
Min Ni, ${ }^{1,2}$ Yiwen Chen, ${ }^{3,4}$ Teng Fei, ${ }^{1,2}$ Dan Li, ${ }^{1,2}$ Elgene Lim, ${ }^{1,2}$ X. Shirley Liu, ${ }^{3,4,5}$ \\ and Myles Brown ${ }^{1,2,5}$ \\ ${ }^{1}$ Division of Molecular and Cellular Oncology, Department of Medical Oncology, Dana-Farber Cancer Institute, Boston, \\ Massachusetts 02215, USA; ${ }^{2}$ Department of Medicine, Brigham and Women's Hospital, Harvard Medical School, Boston, \\ Massachusetts 02215, USA; ${ }^{3}$ Department of Biostatistics and Computational Biology, Dana-Farber Cancer Institute, \\ Boston, Massachusetts 02215, USA; ${ }^{4}$ Harvard School of Public Health, Boston, Massachusetts 02215, USA
}

\begin{abstract}
Androgen-stimulated growth of the molecular apocrine breast cancer subtype is mediated by an androgen receptor (AR)-regulated transcriptional program. However, the molecular details of this AR-centered regulatory network and the roles of other transcription factors that cooperate with AR in the network remain elusive. Here we report a positive feed-forward loop that enhances breast cancer growth involving AR, AR coregulators, and downstream target genes. In the absence of an androgen signal, TCF7L2 interacts with FOXA1 at AR-binding sites and represses the basal expression of AR target genes, including MYC. Direct AR regulation of MYC cooperates with AR-mediated activation of HER2/HER3 signaling. HER2/HER3 signaling increases the transcriptional activity of MYC through phosphorylation of MAD1, leading to increased levels of MYC/MAX heterodimers. MYC in turn reinforces the transcriptional activation of androgen-responsive genes. These results reveal a novel regulatory network in molecular apocrine breast cancers regulated by androgen and AR in which MYC plays a central role as both a key target and a cooperating transcription factor to drive oncogenic growth.
\end{abstract}

[Keywords: breast cancer; cistrome; AR; MYC; TCF7L2; FOXA1]

Supplemental material is available for this article.

Received November 5, 2012; revised version accepted March 6, 2013.

Nuclear receptors are a class of ligand-inducible transcription factors that specifically regulate expression of genes involved in metabolism, development, inflammation, reproduction, and tumorigenesis. In eukaryotic genomes, nuclear receptors interact with coregulators in cooperation with chromatin remodeling factors to control complex regulation of hormone-stimulated gene transcription. Advances in exploring the genome-wide location of estrogen receptor (ER) and androgen receptor (AR) in human solid cancers provide in-depth insights into the role of pioneer transcription factors in cell type-specific regulation of hormone-dependent transcription (Carroll et al. 2005; Lupien et al. 2008).

Pioneer factors have the capacity to interact with condensed chromatin and facilitate chromatin opening, which enables competence for recruitment of other regulatory complexes. FOXA1 is one such pioneer factor required for the functions of diverse nuclear receptors in hormone-driven cancers, such as ER in breast cancer

\footnotetext{
${ }^{5}$ Corresponding authors

E-mail myles_brown@dfci.harvard.edu

E-mail xsliu@jimmy.harvard.edu

Article published online ahead of print. Article and publication date are online at http://www.genesdev.org/cgi/doi/10.1101/gad.209569.112
}

and $\mathrm{AR}$ in prostate cancer, through promoting nuclear receptor-chromatin interactions. Recent genome-wide studies on AR function in breast cancers demonstrated that FOXA1 is a critical coregulator of AR and controls androgen-stimulated gene expression in ER-negative breast cancers that overexpress HER2 (Ni et al. 2011; Robinson et al. 2011). In this breast cancer subtype, termed molecular apocrine breast cancer (Farmer et al. 2005), AR initially activates the canonical Wnt $/ \beta$-catenin pathway by direct induction of $W N T 7 B$ expression and subsequently employs nuclear $\beta$-catenin as a coactivator to stimulate the expression of the secondary class of target genes, including HER3 (Ni et al. 2011). These studies suggest that a hierarchical interaction network of AR and AR-cooperating transcription factors confers differential regulation of an androgen-responsive transcriptional program via a positive feed-forward loop. However, the network of AR cooperating transcription factors and their regulatory roles in the androgen signaling in molecular apocrine breast cancers remain largely unknown.

The AR cistrome is distinct in androgen-dependent breast and prostate cancer cells, although FOXA1 serves as the major determinant for AR recruitment in both cellular contexts (Ni et al. 2011). Comparative investigation of the FOXA1 cistromes in breast and prostate cancer 
cells indicates that the epigenetic status determines the cell type-specific location and function of FOXA1 (Lupien et al. 2008). Studies of global profiling of chromatin structure reveal that FOXA1-binding sites frequently are devoid of DNA methylation (Serandour et al. 2011) and stable nucleosomes (Eeckhoute et al. 2009), and the binding activity of FOXA1 positively correlates with the presence of specific histone modifications marking active enhancers, such as H3K4me1 and H3K4me2 (Lupien et al. 2008). In addition, FOXA1 is required for maintaining active chromatin, thereby providing an optimal platform for recruitment of nuclear receptors and coregulators to execute ligand-stimulated transcriptional activation. In addition to its positive regulatory role, FOXAl is also found to interact with the transducing-like enhancer of slip (TLE)/Groucho corepressor proteins and thereby elicit transcriptional repression (Sekiya and Zaret 2007). Nonetheless, it remains elusive what transcription factors mediate the baseline repression of nuclear receptor target genes to prevent premature gene activation prior to hormone stimulation. In the canonical Wnt signaling pathway, the T-cell factor/lymphoid enhancer factors (TCF/ LEF) are known to recruit TLE/Groucho corepressors and histone deacetylases (HDACs) to create a less accessible chromatin structure, leading to transcriptional repression in the absence of Wnt ligands. We previously observed that TCF7L2 is able to bind the genomic region cooccupied by AR and FOXA1 at the HER3 gene locus prior to androgen stimulation; however, TCF7L2 occupancy is gradually attenuated during androgen exposure ( $\mathrm{Ni}$ et al. 2011). It is unknown whether TCF/LEF factors are involved in FOXA1-mediated transcriptional repression.

To potentiate the proproliferative effect of steroid hormone signaling in cancer, the downstream target genes of the steroid receptors can play both positive and negative roles in modulating the regulatory networks. For example, $A P 2 \gamma$, an estrogen-induced ER target gene, serves as another pioneer factor for ER function (Tan et al. 2011) and also regulates the transcription of the ER gene ESR1 itself (Woodfield et al. 2007). Thus, the function of ER is co-opted to promote the growth of $\mathrm{ER}^{+}$breast cancers. Interestingly, steroid receptors induce similar sets of genes in distinct types of human cancer (Lupien et al. 2008; Robinson et al. 2011), including notably oncogenic transcription factors such as MYC $(c-M y c)$. How relevant the functions of the individual targets are to the steroid receptor regulatory networks in different cancer types is largely unknown.

MYC is one of the most frequently activated oncogenes in a wide variety of human cancers with a broad influence on cell proliferation, survival, differentiation, and genetic stability. Recent genome-wide identification of MYCbinding sites and target genes greatly advanced our understanding of the complex nature of MYC action on gene regulation in tumorigenesis and stem cell differentiation (Li et al. 2003; Zeller et al. 2006; Chen et al. 2008; Kim et al. 2008). It is intriguing that binding of MYC alone is generally insufficient to activate its target genes, and other transcription factors are often required for a full effect on transcription. For example, genes differentially regulated in embryonic stem (ES) cell differentiation tend to be co-occupied by MYC and other stem cell factors (Kim et al. 2010). MYC is transcriptionally up-regulated by ER signaling in $\mathrm{ER}^{+}$breast cancers and regulates the expression of a poor-prognosis cancer signature (Wolfer et al. 2010). Similarly, MYC is highly induced by androgen signaling in molecular apocrine breast cancers. However, the role of MYC and MYC target genes in androgencontrolled breast cancer growth remains unclear.

Here, we report a novel regulatory network in molecular apocrine breast cancers regulated by AR and MYC.

\section{Results}

TCF7L2 and FOXA1 co-occupy androgen-stimulated $A R$-binding sites in molecular apocrine breast cancer cells

Based on our finding of an important role for FOXA1 in facilitating AR action in molecular apocrine breast cancers, we searched for other factors that would functionally interact with FOXA1 in this breast cancer subtype. Analysis of the FOXA1 cistrome in MDA-MB-453 breast cancer cells revealed significant enrichment of the TCF7L2 DNA-binding motif $\left(P<1 \times 10^{-6}\right)$. We therefore tested whether TCF7L2 and FOXA1 proteins physically interact in MDA-MB-453 cells. Coimmunoprecipitation of endogenous proteins (Fig. 1A) suggests that TCF7L2, FOXA1, and AR may cooperate in regulating an androgen-dependent transcriptional network. Of note, we observed that the level of AR coimmunoprecipitated with TCF7L2 or FOXA1 increased upon DHT stimulation (Fig. 1A), likely due to the androgen-induced nuclear accumulation of AR in MDA-MB-453 cells.

TCF7L2 is a member of the TCF/LEF family, a small subset of the high-mobility group (HMG)-box protein family. Like other HMG-box transcription factors, TCF/ LEF factors are devoid of transcriptional activity but are thought to have an architectural function through the modulation of DNA looping and serving as DNA-anchored platforms for recruitment of other transcription factors and chromatin remodeling complexes (Mao and Byers 2011). To further define the functional relationship between TCF7L2, FOXA1, and AR, we determined the TCF7L2 cistrome in MDA-MB-453 breast cancer cells by chromatin immunoprecipitation (ChIP) coupled with DNA sequencing (ChIP-seq). Given that DHT treatment attenuates TCF7L2 recruitment to the AR-FOXA1binding site within the HER3 gene (Ni et al. 2011), we performed the TCF7L2 ChIP-seq in the absence of DHT stimulation. A total of 11,395 high-confidence TCF7L2binding sites were identified $\left(P<1 \times 10^{-12}\right)$. Similar to the AR and FOXA1 cistromes that we previously defined (Ni et al. 2011), the TCF7L2-binding sites were found to be located predominantly at distal intergenic and intronic regions (Supplemental Fig. 1A) and possess highly conserved genomic sequences (Supplemental Fig. 1B). The TCF7L2 cistrome overlaps to a large degree with the FOXA1 cistrome in this cell type over a range of false discovery rate (FDR) cutoffs used for peak calling in the ChIPseq analysis (Fig. 1B; Supplemental Fig. 1C). Specifically, 
A
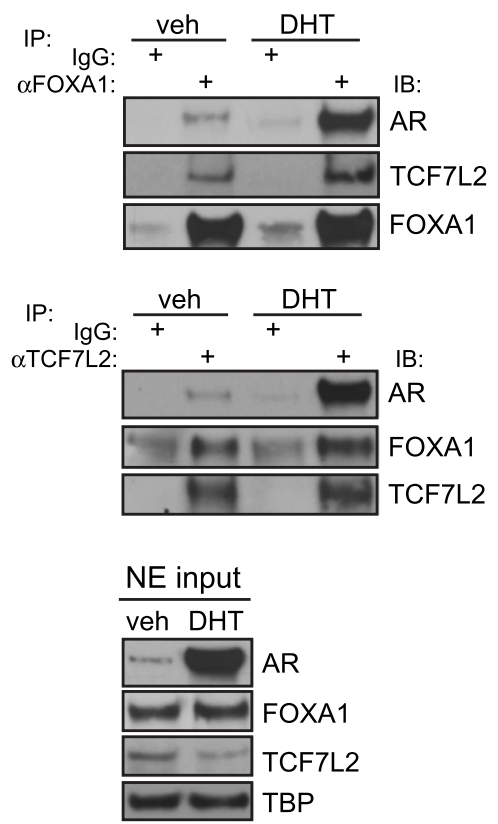

D
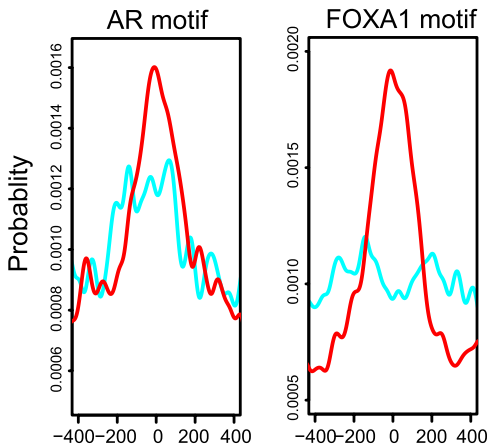

Distance from peak summit (bp)

E

CHARAFE_BREAST_CANCER_LUMINAL_VS_MESENCHYMAL_UP SMID_BREAST_CANCER_BASAL_DOWN CREIGHTON_ENDOCRINE_THERAPY_RESISTANCE_3 CHARAFE_BREAST_CANCER_LUMINAL_VS_BASAL_UP FARMER_BREAST_CANCER_APOCRINE VS LUMINAL DOANE_RESPONSE_TO_ANDROGEN_UP FARMER_BREAST_CANCER_APOCRINE_VS_BASAL SMID_BREAST_CANCER_ERBB2_UP MASSARWEH_TAMOXIFEN_RESISTANCE_UP NELSON_RESPONSE_TO_ANDROGEN_UP CREIGHTON_ENDOCRINE_THERAPY_RESISTANCE_5 DANG_BOUND_BY_MYC

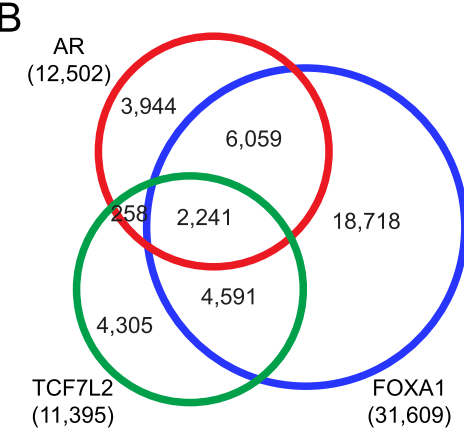

C Average Profiles of TCF7L2 Binding from the Center of Sites
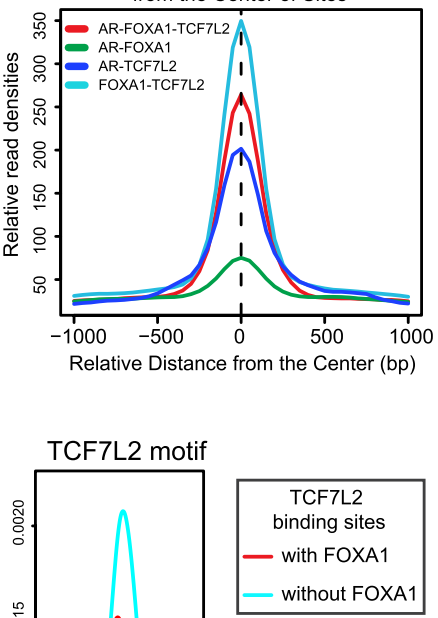
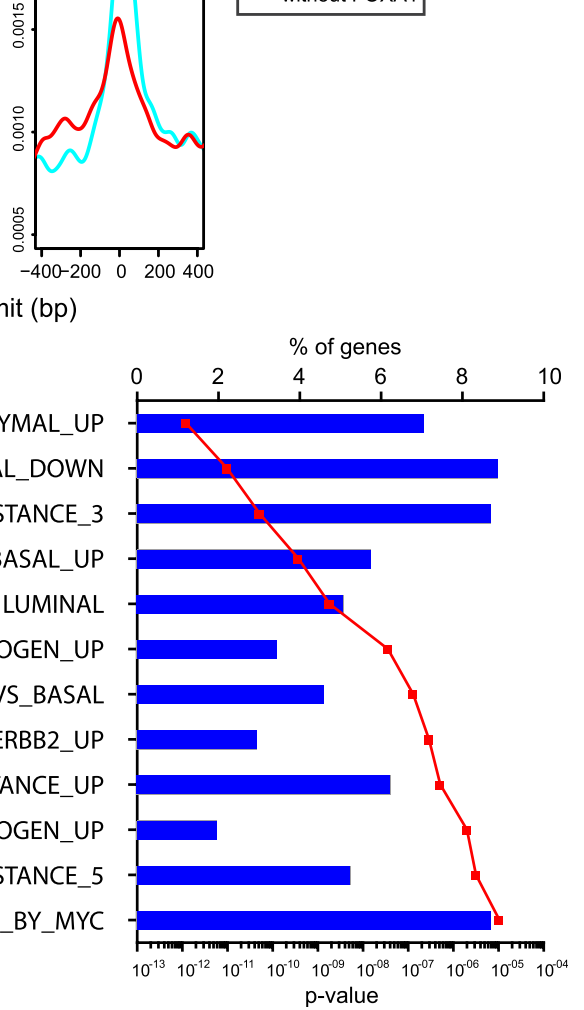

Figure 1. TCF7L2 correlates with AR and FOXA1 in molecular apocrine breast cancers. $(A)$ Endogenous TCF7L2 was coimmunoprecipitated with AR and FOXA1 in MDA-MB-453 breast cancer cells. Nuclear extracts (NEs) from vehicle (veh) or DHT-treated MDAMB-435 cells were immunoprecipitated with antibodies against TCF7L2, FOXA1, or IgG (negative control), respectively. Coprecipitated proteins were analyzed by Western blot using the indicated antibodies. $(B)$ Venn diagram showing the genome-wide overlaps between the TCF7L2, FOXA1, and AR cistromes in MDA-MB-453 cells. (C) ChIP-seq density plot showing the occupancy of TCF7L2 within the $\pm 1-\mathrm{kb}$ regions of the binding sites shared by AR-FOXA1-TCF7L2 (red) or uniquely shared by AR-FOXA1 (green), AR-TCF7L2 (blue), or FOXA1-TCF7L2 (light blue). (D) Enrichment of the binding motifs for AR, FOXA1, or TCF7L2 in the center of the TCF7L2-binding sites with (red) or without (light blue) FOXA1. (E) Functional annotation of the genes with AR-FOXA1-TCF7L2-overlapping sites within $\pm 20 \mathrm{~kb}$ of their TSSs. The top-enriched categories $\left(P<1 \times 10^{-5}\right)$ from GSEA were shown. 
$\sim 30 \%$ of AR-FOXA1-overlapping sites were shared with TCF7L2, and the vast majority of AR-TCF7L2 overlap sites were bound by FOXA1 (Fig. 1B; Supplemental Fig. $1 C)$. In addition, we found that silencing of FOXA1 reduced the amount of AR protein coimmunoprecipitated by TCF7L2 in DHT-treated MDA-MB-453 cells (Supplemental Fig. 2), suggesting that FOXA1 facilitates the interaction between TCF7L2 and AR. Thus, these data reveal a significant potential functional interaction between TCF7L2, FOXA1, and AR on the genome, implicating that the cooperation between TCF7L2 and FOXA1 might modulate the activity of AR in molecular apocrine breast cancer cells. In addition, as the TCF7L2 cistrome was determined in the absence of androgen stimulation, the AR-TCF7L2 and AR-FOXA1-TCF7L2-binding sites are occupied by TCF7L2 in the absence of AR binding.

To better understand the regulatory role of TCF7L2 in AR-regulated gene transcription, we first characterized the sites shared among TCF7L2, FOXA1, and AR (ARFOXA1-TCF7L2 sites) using bioinformatic approaches. By Sitepro analysis (Shin et al. 2009), we compared TCF7L2 ChIP-seq signals between different groups of the TCF7L2 sites that are differentially overlapped with AR and/or FOXA1. We found that TCF7L2 occupancy as determined by ChIP-seq was weaker at the sites with AR occupancy (shown as AR-FOXA1-TCF7L2 and AR-TCF7L2 in Fig. 1C) compared with the TCF7L2 sites without AR binding, such as the sites uniquely overlapped with FOXA1 (shown as FOXA1-TCF7L2 in Fig. 1C). The motif enrichment analysis revealed that the TCF7L2 sites cobound by FOXA1 were enriched for the binding motifs of AR, FOXA1, and TCF7L2, whereas the TCF7L2 sites without FOXA1 occupancy only showed predominant enrichment for the TCF7L2 motif itself (Fig. 1D).

We further characterized the functional annotations of genes potentially coregulated by these three factors using gene set enrichment analysis (GSEA). The genes with AR-FOXA1-TCF7L2-overlapping sites within $\pm 20 \mathrm{~kb}$ of their transcription start sites (TSSs) were compared with the gene sets in the Molecular Signatures Database (MSigDB). We found that these genes were significantly enriched in the overexpression gene signatures of molecular apocrine breast tumors, luminal breast tumors, or HER $2^{+}$breast tumors and also overlapped with androgenup-regulated gene sets from two independent studies (Fig. 1E). Overrepresentation of these gene signatures is consistent with our previous observation of the role of androgen and AR-regulated transcription in the molecular apocrine subtype of breast cancers.

\section{TCF7L2 represses AR-mediated transcriptional activation}

We previously found that FOXA1 is a critical cooperating transcription factor of AR in activating androgen-responsive genes in molecular apocrine breast cancers. To determine the function of the various potential regulatory modules-including AR, FOXA1, and TCF7L2-in androgen signaling, we examined the correlation between binding of the three factors in all possible combinations and DHT-regulated gene expression in MDA-MB-453 breast cancer cells. We observed that binding sites for AR or FOXA1 alone were highly enriched within $\pm 20 \mathrm{~kb}$ of the TSSs of DHT-up-regulated genes (Fig. 2A). Importantly, sites bound by all three factors were the most highly correlated with DHT-induced gene expression (Fig. 2A). While the majority of DHT-up-regulated genes contain binding sites for AR and/or FOXA1 in the vicinity of their TSSs (Fig. 2A,B), sites bound by all three factors are significantly enriched within $\pm 20 \mathrm{~kb}$ of $\sim 20 \%$ of the DHT-up-regulated genes (Fig. 2B). In contrast, the AR-FOXA1-TCF7L2-overlapping sites are not enriched in the vicinity of the genes down-regulated by DHT (Supplemental Fig. 3).

As examples, the AR target genes $K D M 4 B$ and $A Q P 3$ have AR-FOXA1-TCF7L2-overlapping sites within $\pm 20 \mathrm{~kb}$ of their TSSs (Fig. 2C). Inhibition of AR by shRNA attenuated DHT-mediated up-regulation of $K D M 4 B$, $A Q P 3$, and other AR target genes, and importantly, inhibition of TCF7L2 increased their expression even in the absence of DHT stimulation (Fig. 2D; Supplemental Fig. 4). Consistent with the changes on mRNA levels, knockdown of TCF7L2 by three individual shRNAs substantially enhanced AR and FOXA1 binding to their overlapping sites in the KDM $4 B$ and $A Q P 3$ genes (Fig. $2 E)$. The protein levels of AR and FOXA1 were not significantly affected by TCF7L2 depletion (Fig. 2F). These data indicate that preoccupancy of TCF7L2 confers basal repression of the expression of AR target genes prior to androgen stimulation and dampens AR-mediated transcriptional activation. Consistently, Sitepro analysis revealed that global AR binding at the AR-TCF7L2 or AR-FOXA1-TCF7L2 sites was weaker than at the ARFOXA1 sites lacking TCF7L2 binding (Fig. 2G). However, the strongest binding of FOXA1 was observed at the AR-FOXA1-TCF7L2 sites, especially compared with AR-FOXA1 sites (Supplemental Fig. 5). While the TCF7L2 cistrome derived from DHT-treated MDA-MB-453 cells shows highly conserved genomic sequences (Supplemental Fig. 6A), DHT treatment greatly decreased the binding intensity of TCF7L2 at the FOXA1 and/or AR sites that are occupied by TCF7L2 before stimulation (Supplemental Fig. 6B). Functionally, the DHT-up-regulated genes with AR-FOXA1-TCF7L2 sites or AR-FOXA1 sites are involved in different biological processes (Supplemental Fig. 7). Consistent with the known function of TCF7L2 in the Wnt signaling pathway, the TCF7L2-bound genes in DHT-treated cells are enriched in various cancer pathways, including consensus Wnt signaling genes (Supplemental Fig. 6C). Collectively, these results suggest that while FOXA1 creates a primed state for efficient AR recruitment, TCF7L2 represses the baseline and androgeninduced expression of AR target genes.

\section{Cooperation of AR, FOXA1, and TCF7L2 controls androgen-induced MYC expression}

By defining the DHT-up-regulated transcriptional program in MDA-MB-453 breast cancer cells, we observed a significant enrichment of genes involved in the Wnt 
A

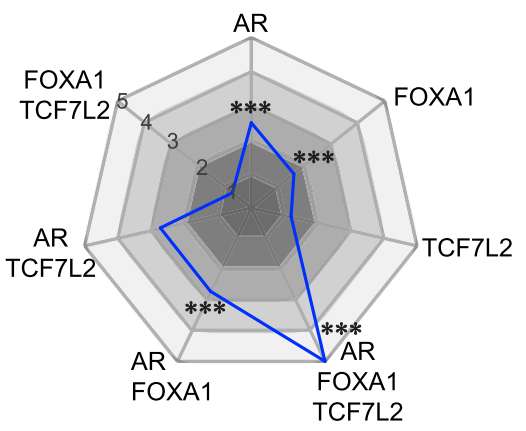

B

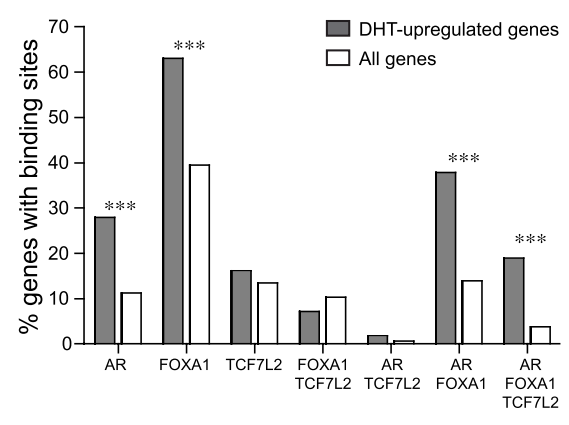

D
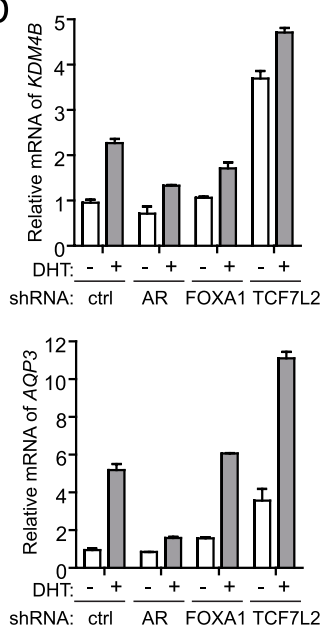

$E$
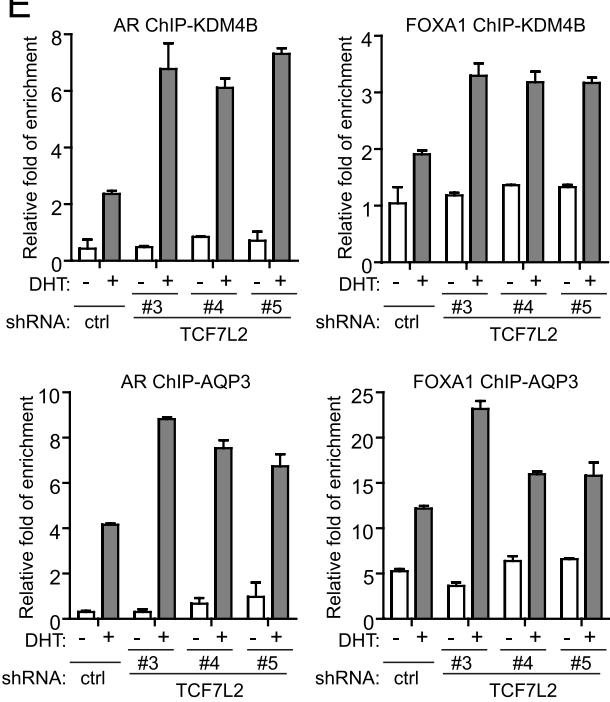
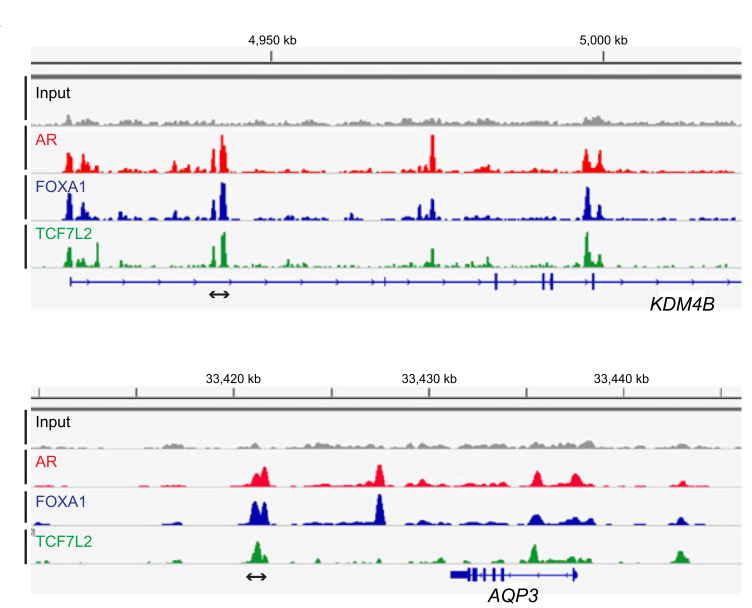

$\mathrm{F}$
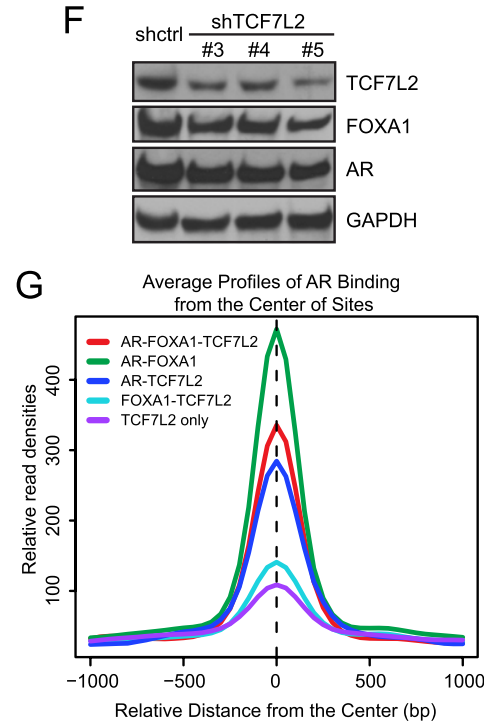

Figure 2. TCF7L2 collaborates with AR and FOXA1 in the control of DHT-mediated transcriptional activation. $(A, B)$ Correlation between DHT-up-regulated gene expression and the binding sites of AR, FOXA1, and TCF7L2 in all possible combinations. $(A)$ The fold enrichment was determined by normalizing the percentage of DHT-up-regulated genes at the 16-h time point with the binding sites in the given category within $\pm 20 \mathrm{~kb}$ of their TSSs to the percentage of all genes with the same type of binding sites within $\pm 20 \mathrm{~kb}$ of their TSSs. The values of fold enrichment were plotted in a radar chart. $\left.{ }^{\star \star \star}\right) P<1 \times 10^{-6}$. $(B)$ The fraction of genes with the binding sites of AR, FOXA1, and TCF7L 2 in the indicated combination was plotted. The absolute numbers of DHT-up-regulated genes at $16 \mathrm{~h}$ in each category are AR, 31; FOXA1, 70; TCF7L2, 18; FOXA1-TCF7L2, eight; AR-TCF7L2, two; AR-FOXA1, 42; and AR-FOXA1-TCF7L2, 21. (C) AR, FOXA1, and TCF7L2 occupancy around two representative AR target genes: KDM4B and AQP3. The black segments with double arrowheads show the binding sites validated by direct ChIP-qPCR. (D) Expression of KDM4B and AQP3 was monitored by quantitative real-time RT-PCR in MDA-MB-453 cells transduced with the indicated lentiviral shRNAs followed by treatment with vehicle or DHT for $6 \mathrm{~h}$. mRNA levels are shown as means with standard deviation (SD) from three replicates. (E) MDA-MB-453 cells were transduced with control lentiviral shRNA or three different TCF7L2 shRNAs followed by DHT treatment for $6 \mathrm{~h}$. The binding of AR and FOXA1 to the $K D M 4 B$ or $A Q P 3$ gene loci was analyzed by ChIP-qPCR. (F) Expression of TCF7L2, AR, and FOXA1 proteins was analyzed by Western blot in MDA-MB453 cells transduced with the control or TCF7L2 lentiviral shRNAs. (G) Density plot of AR ChIP-seq signals within \pm 1 -kb regions of the binding sites shared by AR-FOXA1-TCF7L2, shared by only two factors, or unique to TCF7L2, respectively. 
signaling pathway (Ni et al. 2011). Screening of these overexpressed Wnt pathway genes revealed that MYC is the top-ranked gene markedly induced by DHT at the early time point. Analysis of the AR, FOXAl, and TCF7L2 cistromes identified two regions with ARFOXA1-TCF7L2-overlapping sites located $\sim 67 \mathrm{~kb}$ and $340 \mathrm{~kb}$ upstream of the TSS of the MYC gene (labeled as Enh1 and Enh2 in Fig. 3A). Of note, the $-340-\mathrm{kb}$ site contains the 8q24 risk single-nucleotide polymorphism (SNP) associated with colorectal cancer risk and overlaps with a distal enhancer of the $M Y C$ gene occupied by TCF7L2 in colorectal cancer cells (Pomerantz et al. 2009). Consistent with their putative enhancer function, the interaction between the MYC promoter and the two distal AR-FOXA1-TCF7L2-binding regions (Enh1 and Enh2) was detected by chromosome conformation capture (3C) (Pomerantz et al. 2009) at a significantly higher frequency than other regions within the MYC locus in MDA-MB-453 cells by DHT stimulation, and the looping between the MYC promoter and the Enh2, but not the Enh1, region was highly induced by DHT compared with vehicle treatment (Fig. 3B). Over the course of DHT treatment, AR occupancy was gradually and significantly increased at Enh1 and three subpeaks within the Enh2 region (Enh-2a, Enh-2b, and Enh-2c) (Fig. 3C), and MYC mRNA and protein expression was rapidly and substantially induced (Fig. 3D,E). In contrast, TCF7L2 binding gradually decreased at the MYC enhancers (Fig. 3C). Notably, FOXA1 resided at these two enhancers prior to DHT-induced AR activation and remained associated during the time course (Fig. 3C). Silencing of FOXA1 impaired the interaction of the MYC promoter with two enhancer regions (Fig. 3F), leading to repression of the DHT-mediated MYC up-regulation (Fig. 3G). Conversely, inhibition of TCF7L2 significantly elevated MYC expression in either vehicle or DHT-treated MDA-MB-453 cells (Fig. 3G), possibly due to increased interactions between the MYC promoter with distal enhancers in TCF7L2 knockdown cells (Fig. 3F). These results suggest that the preoccupancy of TCF7L2 at the MYC enhancers represses the promoter-enhancer interaction and maintains $M Y C$ expression at basal levels and that continuous FOXA1 binding facilities AR recruitment, leading to derepression of MYC with loss of TCF7L2 binding and further activation of $M Y C$.

\section{Genome-wide identification of androgen-stimulated MYC target sites in molecular apocrine breast cancer cells}

To investigate the role of MYC in androgen signaling, we performed ChIP-seq analysis to define the MYC cistrome in MDA-MB-453 breast cancer cells. We identified $\sim 7000$ high-confidence MYC-binding sites after $6 \mathrm{~h}$ of DHT stimulation $\left(P<1 \times 10^{-12}\right)$. The vast majority of MYCbinding sites was found at gene-proximal promoters and gene bodies (Fig. 4A), and the global MYC binding is predominantly enriched at the gene TSS (Fig. 4B). Motif analysis revealed a significant enrichment of the E-box motif of MYC:MAX in the DHT-induced MYC cistrome specific to MDA-MB-453 cells (Fig. 4B, inserted panel). It has been shown that the MYC regulatory network is active and comparable in ES cells and various cancers (Kim et al. 2010). Similarly, we observed that a large proportion of the MYC-binding sites were overlapped between MDA-MB-453 breast cancer cells and other cell types using publically available ENCODE data, including H1 human ES (hES) cells and K562 chronic myelogenous leukemia (CML) cells (Fig. 4C). In addition, the MYCbound genes are also highly similar between MDA-MB453 and H1 hES cells (Fig. 4D). MYC is an important transcriptional regulator in various aspects of cellular function, including replication, growth, metabolism, differentiation, and apoptosis. Consistent with its known function, gene annotation analysis revealed that MYCbound genes in MDA-MB-453 cells were primarily associated with regulation of translation and cell cycle progression (Fig. 4E,F).

\section{MYC occupancy is associated with AR-mediated transcriptional activation in molecular apocrine breast cancer}

To investigate the role of MYC in the androgen signaling pathway in molecular apocrine breast cancers, we assessed the correlation between MYC binding and androgen-regulated gene expression in MDA-MB-453 cells. We found that MYC occupancy is significantly enriched at the proximal promoter regions ( $\pm 2 \mathrm{~kb}$ of TSSs) of DHT-up-regulated genes $\left(P<7.9 \times 10^{-6}\right)$ (Fig. 5A). Moreover, MYC binding at the promoters of the DHT-upregulated AR target genes or all DHT-up-regulated genes was stronger than at the promoters of all genes (Fig. 5B). Strikingly, $\sim 76 \%$ of DHT-induced MYC target genes were also direct AR targets $(P<0.0036)$ (Fig. 5C). By defining the DHT-up-regulated MYC targets in MDAMB-453 breast cancer cells, we determined that these genes were significantly enriched in the overexpressed gene signatures of $\mathrm{ER}^{+}$or HER2 ${ }^{+}$breast tumors (Fig. 5D) using Oncomine Concepts Map analysis (Rhodes et al. 2007). These gene signatures were also overrepresented in the AR target genes, as defined in our previous study (Ni et al. 2011). Moreover, this MYC target gene set is also correlated with a more malignant or invasive state of breast cancers, such as the gene signatures from highgrade and metastatic breast tumors as well as triplenegative breast tumors (Fig. 5D). Given the recent findings that MYC is involved in amplification of existing transcriptional programs in different cellular contexts (Lin et al. 2012; Nie et al. 2012), our study suggests that AR may employ its target gene MYC to amplify the androgen-regulated downstream gene expression program in molecular apocrine breast cancers.

The androgen signaling pathway enhances the activity of MYC in molecular apocrine breast cancer

The transcriptional activity of MYC is entirely dependent on dimerization with MAX, an abundant and ubiquitously expressed basic helix-loop-helix leucine zipper (b-HLH-LZip) protein. However, the availability of MAX 
Ni et al.

A

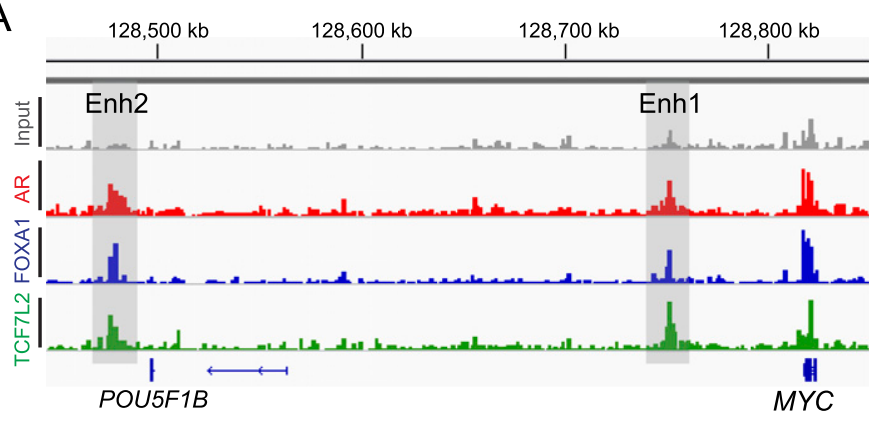

C
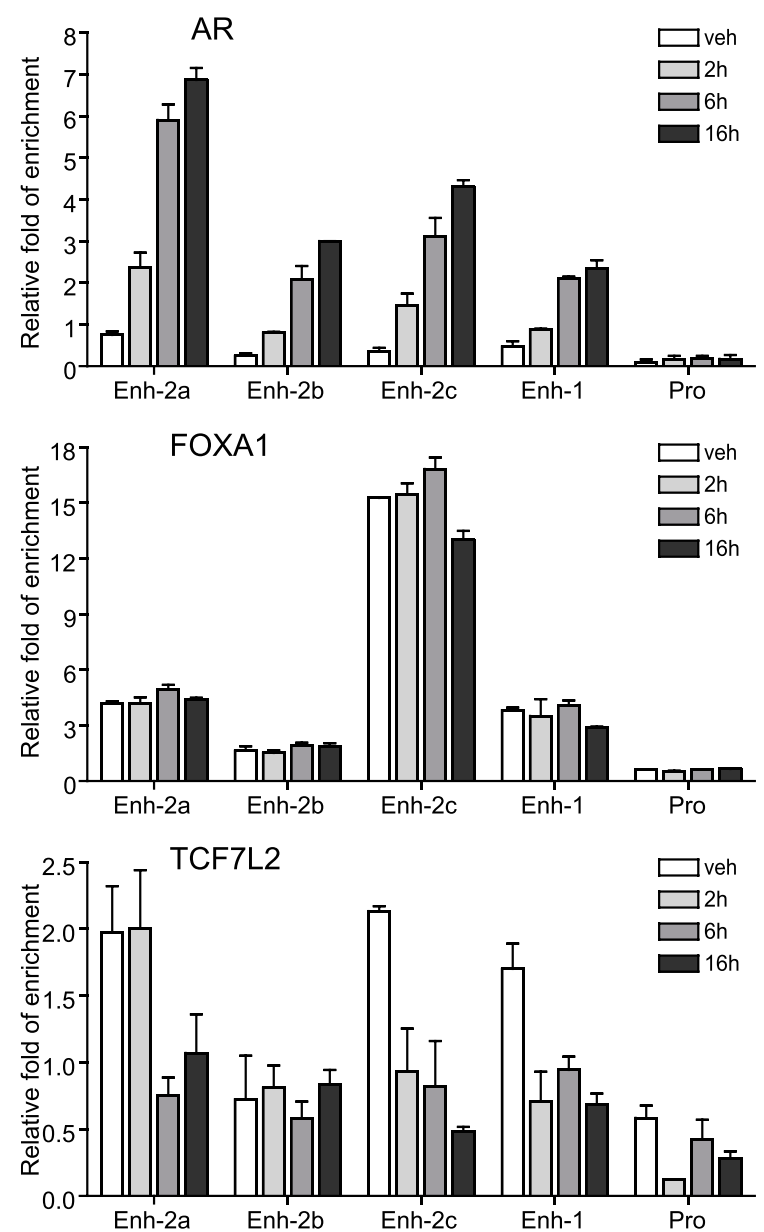

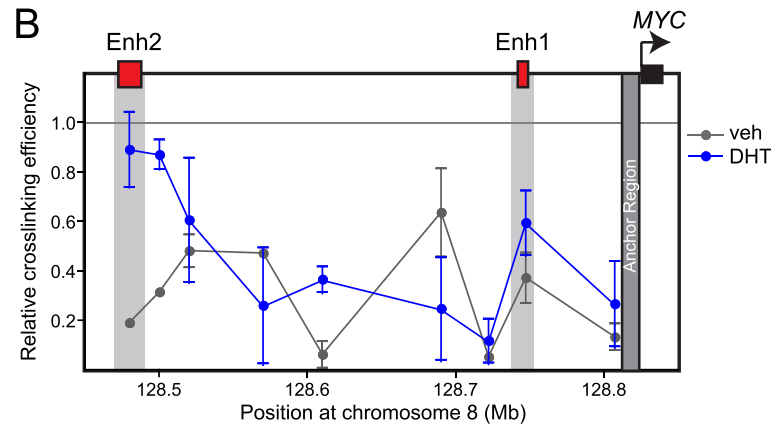

$E$

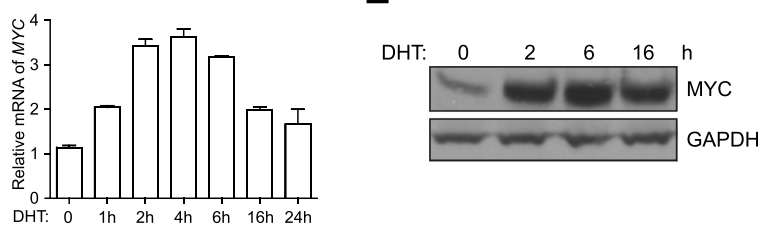

$\mathrm{F}$

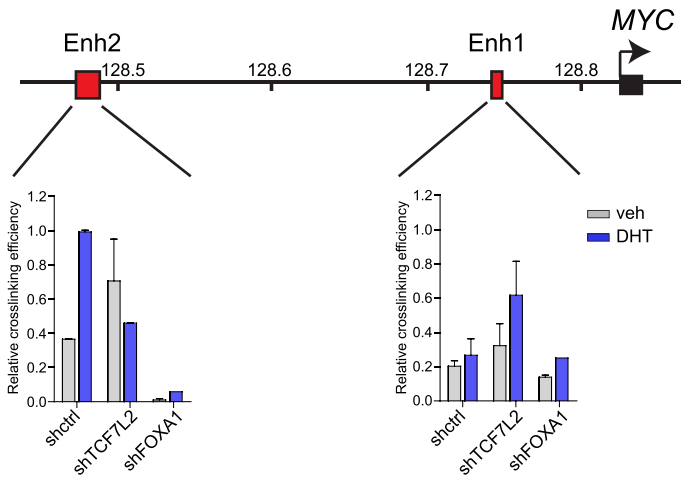

G

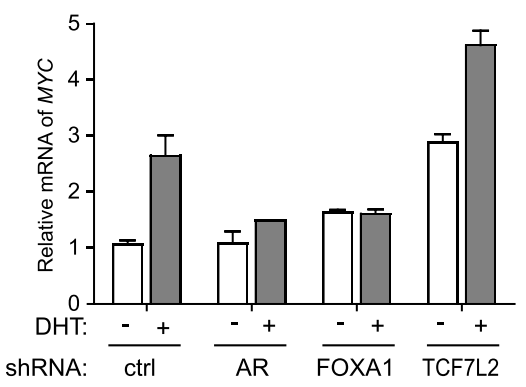

Figure 3. Cooperation of AR, FOXA1, and TCF7L2 regulates the transcriptional activation of MYC. $(A)$ Schematic graph shows the AR-FOXA1-TCF7L2 sites (shaded bars) within the MYC gene locus as defined by ChIP-seq in MDA-MB-453 cells. (B) 3C analysis within the MYC gene locus harboring AR-FOXA1-TCF7L2 sites as shown in A. The relative cross-linking frequency between the anchor region (dark-gray bar) and distal fragments (shaded bars) was determined by qPCR and normalized to the control region. $(C)$ ChIP-qPCR analysis of AR, FOXA1, and TCF7L2 binding at the MYC gene locus in MDA-MB453 cells treated with vehicle (veh) or DHT for the indicated time course. Primers are designed specifically for the promoter region (Pro), the enhancer 1 region (Enh-1), and the three subpeaks within enhancer 2 (Enh-2a, Enh-2b, and Enh-2c). $(D)$ Expression of MYC mRNA was determined by real-time RTPCR in MDA-MB-453 cells treated with DHT for the indicated time course. (E) Expression of MYC proteins was determined by Western blot in the DHT-treated MDA-MB-453 cells. $(F)$ The relative cross-linking frequency between the anchor region and the two enhanced regions of the MYC gene was assessed by 3C analysis in MDA-MB-453 cells with shRNA knockdown of TCF7L2 or FOXA1 followed by vehicle or DHT treatment for $4 \mathrm{~h}$. (G) Expression of MYC mRNA was determined by real-time RT-PCR in MDA-MB-453 cells transduced with lentiviral shRNAs for AR, FOXA1, TCF7L2 or control with vehicle or DHT treatment for $6 \mathrm{~h}$.

to MYC is profoundly dependent on the expression level of MAD1 (Ayer et al. 1993). It is known that the PI3K/AKT signaling pathway promotes the degradation of MAD1 protein by phosphorylation of MAD1, which in turn enhances the transcriptional activity of MYC (Zhu et al. 2008; Chou et al. 2009). 
A

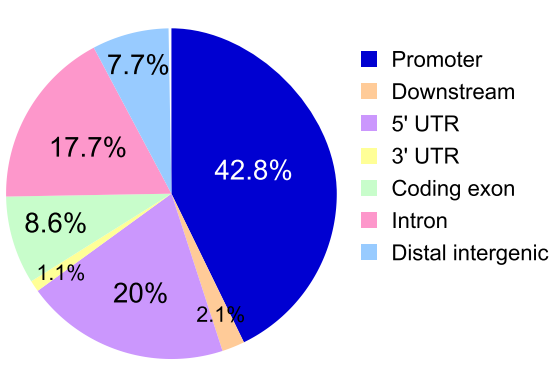

C

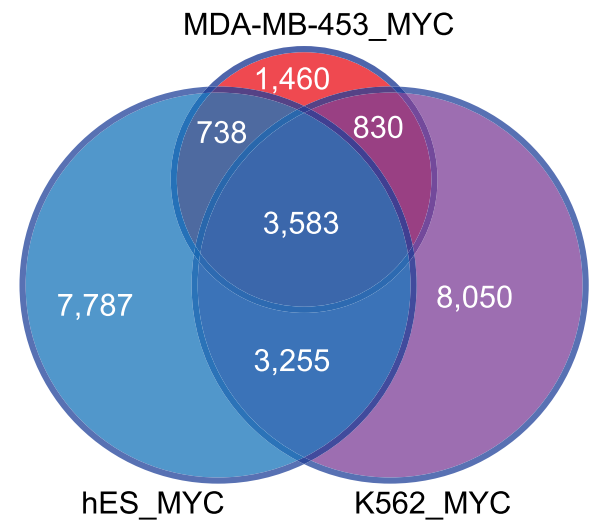

$\mathrm{E}$

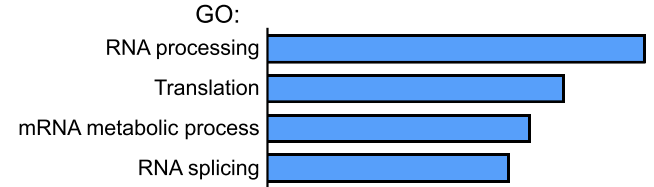

Macromolecule catabolic process

Translational elongation

Ribonucleoprotein biogenesis

DNA metabolic process

Cell cycle
Transcription

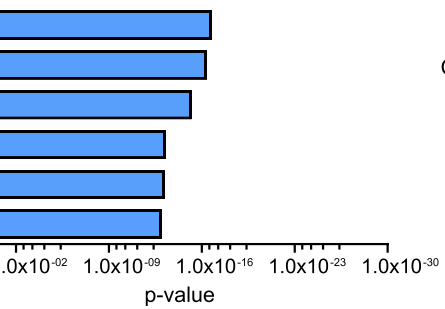

$\mathrm{B}$
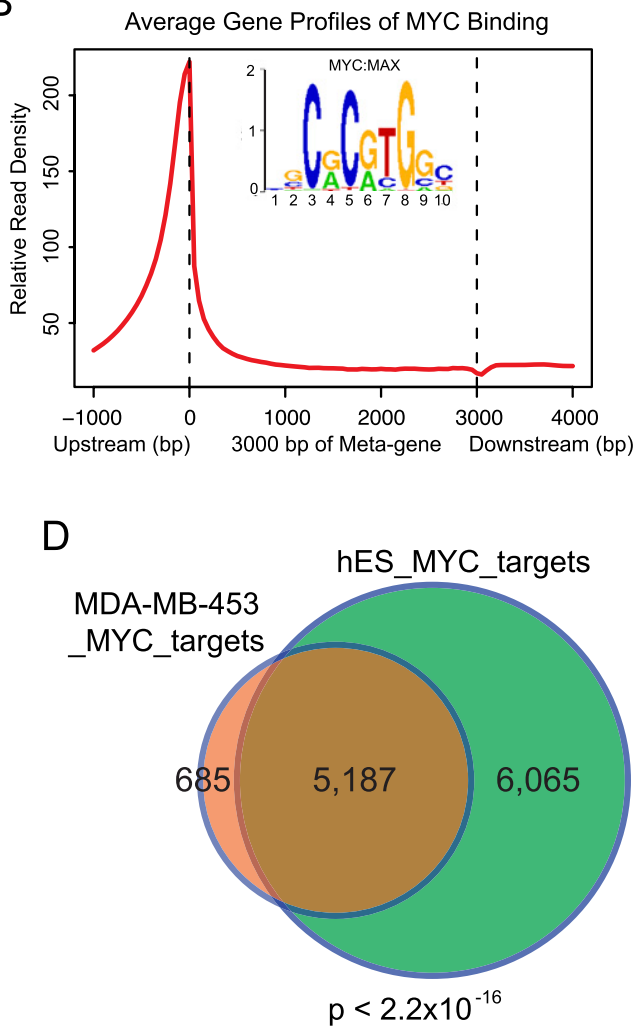

$\mathrm{F}$

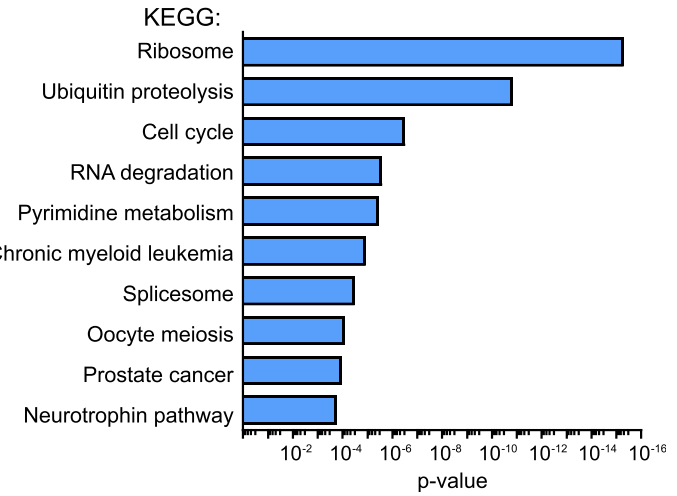

Figure 4. Characterization of a DHT-stimulated MYC cistrome. (A) Genome-wide distribution of DHT-stimulated MYC-binding sites specific to MDA-MB-453 breast cancer cells. $(B)$ The distribution of MYC ChIP-seq signals around human metagenes. The E-box motif of MYC:MAX enriched in the MYC cistrome is shown in the insert. $(C)$ Venn diagram showing the overlaps between the MYC cistromes specific to DHT-stimulated MDA-MB-453 breast cancer cells, H1 hES cells, and K562 human CML cells. (D) Venn diagram showing overlaps between MYC-bound targets in MDA-MB-453 and H1 hES cells. $(E, F)$ Functional annotation analysis of MYC-bound targets in MDA-MB-453 breast cancer cells. The top enriched terms of gene ontology (GO) $(E)$ and KEGG pathways $(F)$ ranked by $P$-values are shown.

In MDA-MB453 cells, we observed that activation of the HER2/HER3 and PI3K/AKT signaling cascade by DHT led to increased phosphorylation of MAD1, resulting in a substantial decrease in total MAD1 protein (Fig. 6A). Specific inhibition of AR function by the AR antagonist bicalutamide or of the androgen-induced signaling cascade by the HER2 inhibitor lapatinib or Wnt inhibitor IWP2 prevented phosphorylation and degradation of MAD1 and induction of MYC as well (Fig. 6B,C). Consequently, while the interaction between MAX and MYC was significantly increased following DHT treatment, their dimerization was markedly abrogated by addition of AR or HER2 inhibitors (Fig. 6D). To assess the effect of inhibition of the androgen signaling pathway on the transcriptional activity of MYC, we examined the recruitment of MYC and RNA polymerase II (pol II) to the promoters of several 
Ni et al.

A

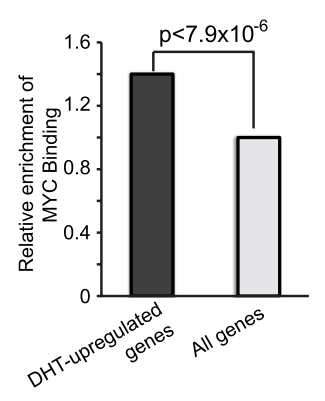

B

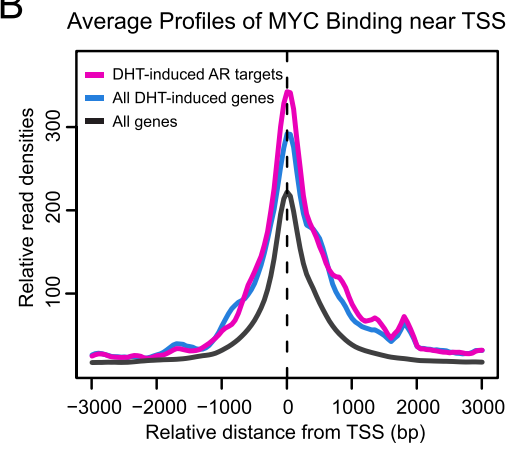

C

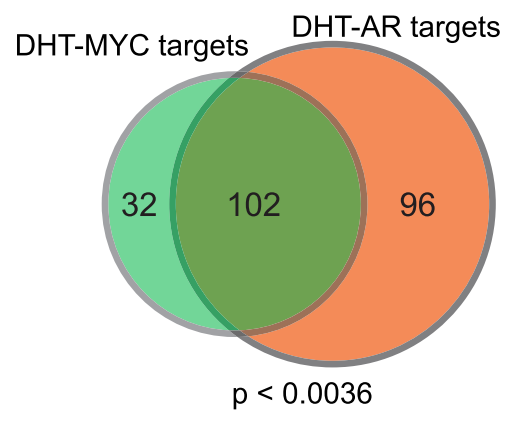

D
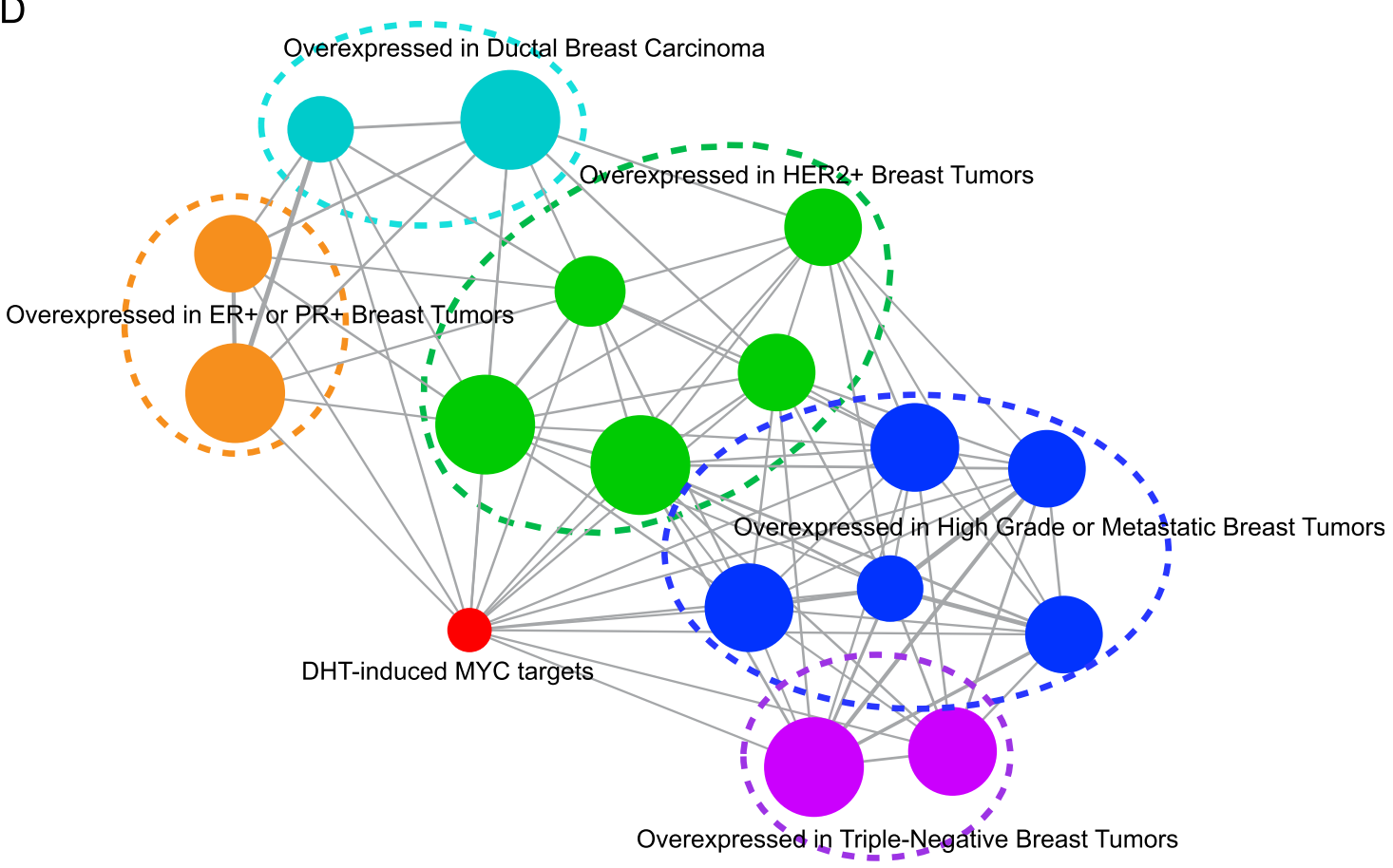

Figure 5. MYC occupancy is associated with AR-mediated transcriptional up-regulation in molecular apocrine breast cancers. $(A)$ Correlation between DHT-up-regulated gene expression and MYC occupancy. $P$-value is shown. (B) The ChIP-seq density plot is shown for MYC binding around the TSSs of DHT-up-regulated AR target genes (pink), all DHT-up-regulated genes (blue), and all genes (black), respectively. $(C)$ Venn diagram showing the overlaps between DHT-up-regulated MYC and AR target genes in MDA-MB-453 cells. (D) An enrichment network view showing the correlation between DHT-up-regulated MYC target genes and gene expression signatures from primary breast tumors. The DHT-induced MYC target genes were analyzed by Oncomine Concepts analysis to compare with all published gene signatures from breast cancer patient samples. Each node represents a gene set or molecular concept, and significantly associated sets $\left(q<1 \times 10^{-5}\right)$ were linked by an edge. The size of a node is proportional to the number of genes in the corresponding gene set, and the thickness of the edge is proportional to the number of overlapped genes between the linked gene sets. Enriched molecular concepts were grouped into five clusters as indicated by the rings with distinct colors.

MYC target genes by ChIP and quantitative PCR (ChIPqPCR) analysis. We observed that DHT stimulation promoted MYC binding with concomitant increased occupancy of pol II at the proximal promoters of DHT-induced MYC targets, including not only bona fide MYC targets, such as JUN and VEGFA, but also many AR target genes (Fig. 6E). Upon administration of bicalutamide or lapatinib, MYC and pol II recruitment was significantly impaired (Fig. 6E). These data suggest that the AR-mediated activation of the HER2 signaling cascade cooperates with direct AR-mediated induction of MYC expression to enhance MYC action in molecular apocrine breast cancers.
MYC enforces androgen-induced transcriptional activation in molecular apocrine breast cancer cells

To investigate the role of MYC in androgen-regulated gene transcription, we performed an RNA sequencing (RNA-seq) analysis to identify differentially expressed genes upon silencing of MYC in MDA-MB-453 cells. Given that MYC activation increases total RNA levels (Lin et al. 2012), we chose an early time point (6 h) for DHT treatment and confirmed that the levels of mRNA and total RNA were unchanged (Supplemental Fig. 8A), whereas MYC expression was already induced at this 
A

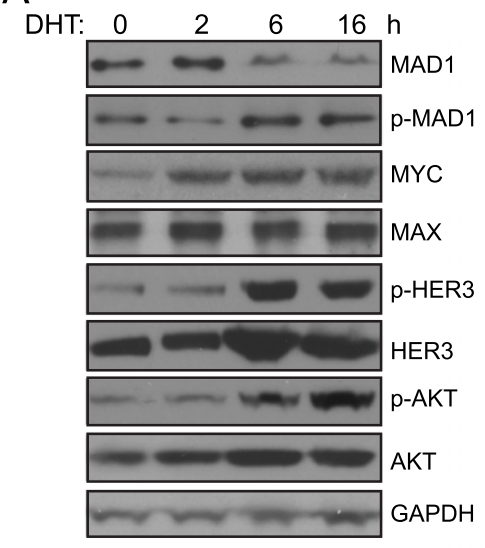

B

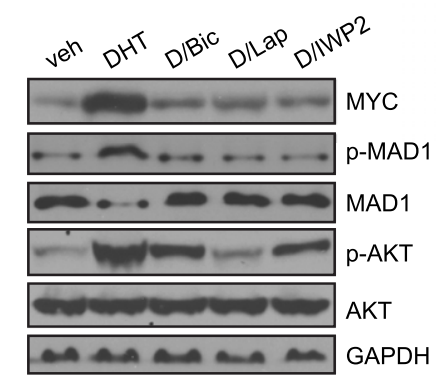

C

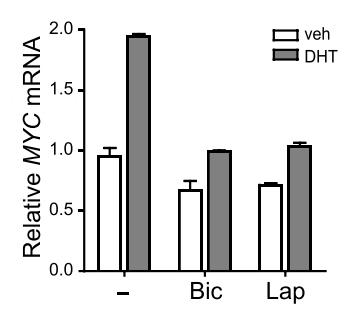

$\mathrm{E}$
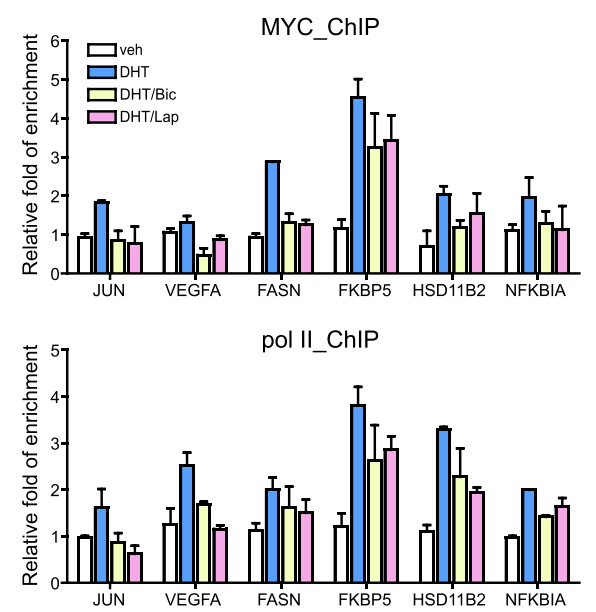

Figure 6. The transcriptional activity of MYC is enhanced by androgen signaling in molecular apocrine breast cancer cells. $(A)$ DHT treatment activates the HER2/HER3 signaling pathway, leading to increased phosphorylation of MAD1 and decreased expression of total MAD1 proteins. After treatment with DHT for the indicated time points, MDA-MB-453 cells were subjected to Western blot analysis for the indicated proteins. (B) MDA-MB-453 cells were treated with vehicle (veh), DHT, or DHT together with $2 \mu M$ of bicalutamide (Bic), $100 \mathrm{nM}$ lapatinib (Lap), or $1 \mu \mathrm{M}$ IWP2 for $6 \mathrm{~h}$ and assayed for expression of the indicated proteins. (C) MYC mRNA was determined by real-time RT-PCR in MDA-MB-453 cells treated with bicalutamide or lapatinib in the presence or absence of DHT for $6 \mathrm{~h}$. (D) The physical interaction between endogenous MYC and MAX proteins were assessed by coimmunoprecipitation assays in the nuclear extracts of MDA-MB-453 cells treated with the indicated compounds for $6 \mathrm{~h}$. The nuclear extracts and the copurified proteins were analyzed by Western blot. (E) MYC and pol II binding to promoters of the MYC target genes was examined by ChIP-qPCR in MDA-MB-453 cells treated with DHT alone or together with bicalutamide or lapatinib for $6 \mathrm{~h}$.

time point (Fig. 3E). Comparing the DHT-regulated transcriptomes between the control and siMYC conditions revealed that MYC knockdown diminished the induction of $\sim 50 \%$ of the DHT-up-regulated genes (Fig. 7A). Of note, $\sim 70 \%$ of these genes are direct MYC targets with promoter occupancy by MYC, half of which are also direct AR targets (Fig. 7B, shown as siCtrl-only DHT-Up genes). Direct ChIP-qPCR experiments demonstrated that the promoter occupancy of pol II at these DHT-upregulated MYC targets was markedly increased in response to DHT but was significantly attenuated by MYC knockdown (Fig. 7C). Meanwhile, the recruitment of AR to the genes targeted by both AR and MYC was unchanged or only slightly affected by MYC silencing (Fig. 7D). In addition, within the DHT-stimulated genes whose activation is unaffected by MYC silencing, $\sim 78 \%$ are $A R$ targets, although MYC binding was observed at the promoters of $>60 \%$ of these AR target genes (Fig. $7 \mathrm{~B}$, shown as Overlap DHT-Up_genes). For this group of genes, silencing of MYC had little effect on DHT-induced recruitment of pol II to their promoters (Fig. 7C), and the increase of AR occupancy by DHT stimulation was unaffected or slightly enhanced (Fig. 7D). Although MYC expression is not completely depleted in MDA-MB-453 cells, we speculate that the transcriptional activation of this group of DHT-up-regulated genes is more dependent on $\mathrm{AR}$, compared with those genes directly repressed by MYC silencing. For the DHT-repressed gene expression, we observed distinct gene sets between control and siMYC conditions in MDA-MB-453 cells (Fig. 7A). Interestingly $\sim 70 \%$ of the DHT-down-regulated genes specific to MYC silencing also have MYC-binding sites within their promoters (Supplemental Fig. 8B), suggesting that expression of these genes also depends on MYC. Taken together, these results demonstrate that MYC acts as a key target and a downstream effector of AR to reinforce androgen signaling in molecular apocrine breast cancer cells.

\section{Discussion}

As a prominent nuclear receptor driving prostate tumorigenesis, AR is emerging as a proproliferative transcription factor in a subgroup of ER-negative breast cancers 
Ni et al.

A
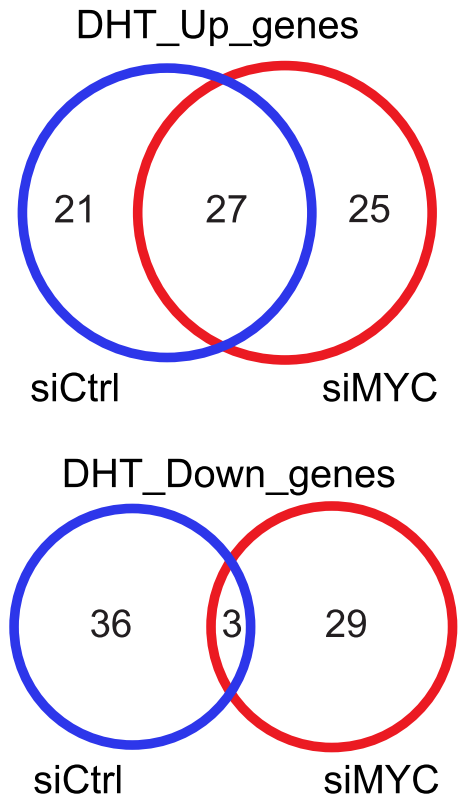

B

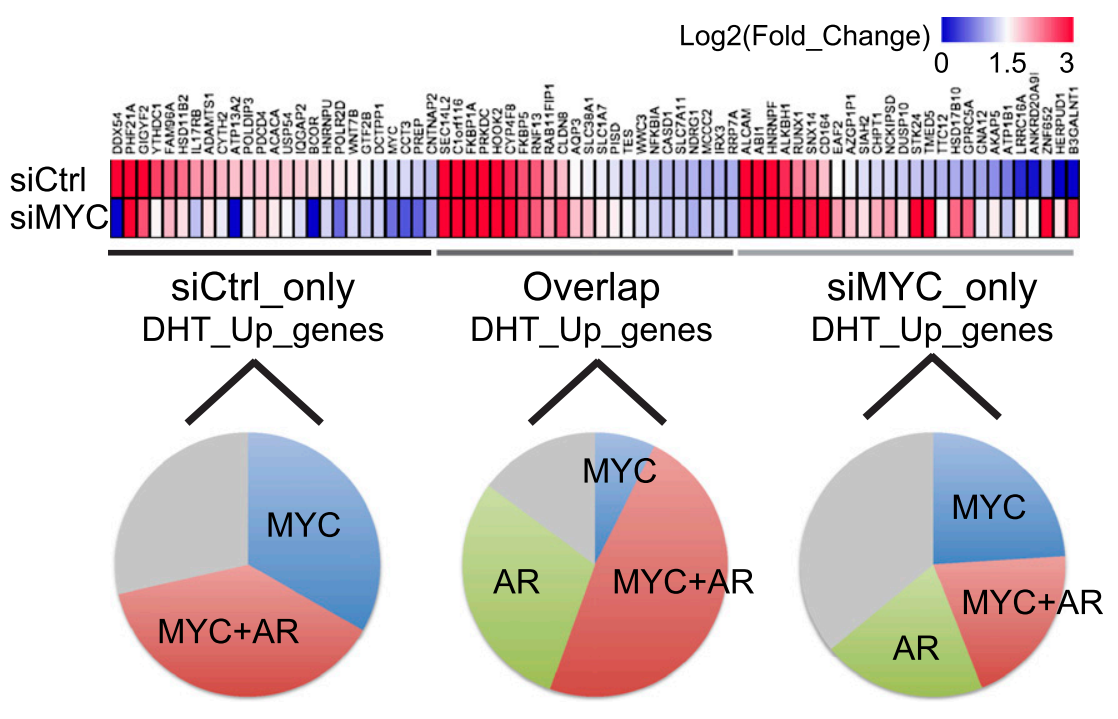

C

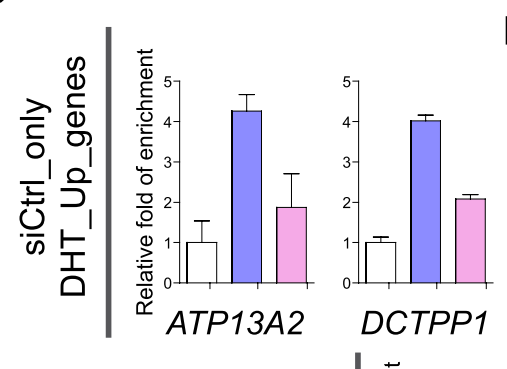

pol II ChIP

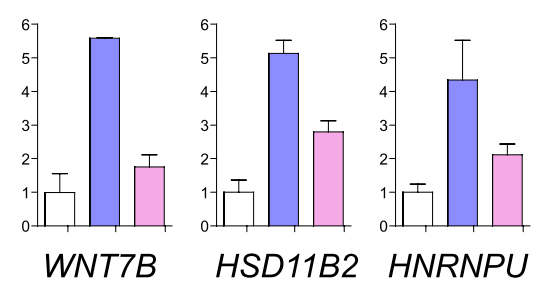

D
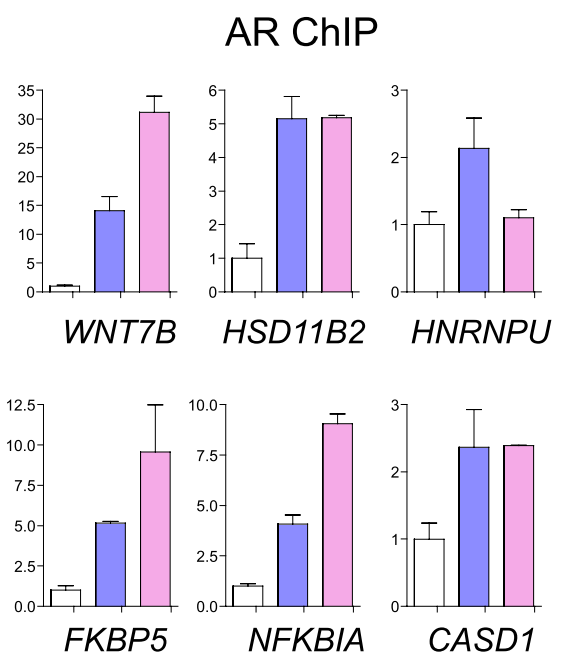

$\square$ siCtrl-veh
$\square$ siCtrl-DHT
$\square$ siMYC-DHT

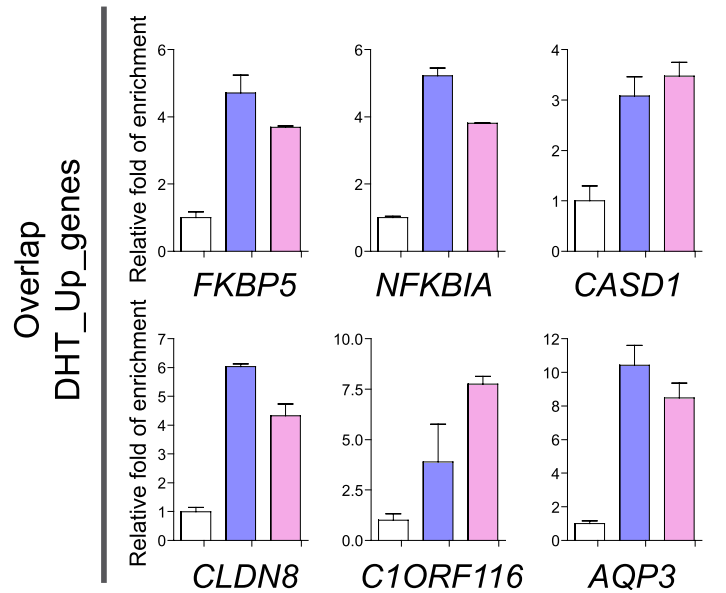

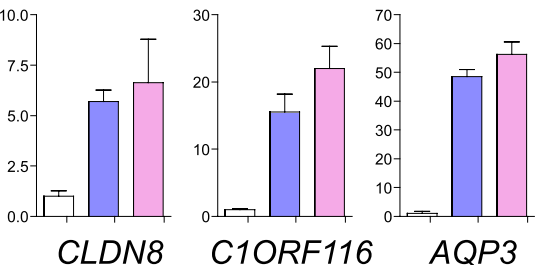

Figure 7. MYC reinforces androgen-induced transcriptional activation. $(A)$ Venn diagrams showing overlaps of the DHT-regulated genes between control siRNA (siCtrl) and siMYC transfected MDA-MB-453 cells. The differential DHT-regulated genes were defined by comparing the expression profile of siCtrl-DHT or siMYC-DHT with the profile of siCtrl-vehicle, respectively. $(B)$ Heat map showing $\log 2$ fold change in the DHT-induced gene expression of siCtrl and siMYC conditions, and pie graphs showing a fraction of the genes in each category with binding of AR ( $\pm 20 \mathrm{~kb}$ from the TSS) and/or MYC ( $\pm 2 \mathrm{~kb}$ from the TSS). (C,D) Direct ChIP-qPCR was performed to examine the recruitment of pol II to the promoters $(C)$ and AR to its target sites $(D)$ of the indicated DHT-up-regulated genes in MDAMB-453 cells transfected with siCtrl or siMYC followed by DHT treatment.

that overexpresses the oncogene HER2, termed the molecular apocrine subtype. Our prior work revealed an AR-regulated signaling cascade involving the primary induction of $W N T 7 B$ for activation of the Wnt $/ \beta$-catenin pathway and the secondary response of AR- $\beta$-cateninmediated HER3 up-regulation to activate the HER2 
signaling pathway (Ni et al. 2011). While increasing evidence suggests that the AR pathway is under the control of other signaling pathways in molecular apocrine breast cancers (for review, see Lim et al. 2012), we identified a novel positive feed-forward mechanism in which AR actives MYC, which in turn reinforces the androgenstimulated transcriptional program by acting on the promoters of $\sim 70 \%$ of androgen-responsive genes, including direct AR targets (Fig. 8).

Transcriptional activation of $M Y C$ represents a very early response and is tightly controlled by AR in collaboration with FOXA1 and TCF7L2. FOXA1 is a pioneer transcription factor of AR in governing androgen-regulated transcription in molecular apocrine breast cancers. We showed that TCF7L2 physically interacts with FOXA1 and mediates the transcriptional repression of specific AR target genes, including MYC. Prior to hormone stimulation, TCF7L2 represses the expression of AR target genes to a low baseline level, and concomitant FOXA1 occupancy presumably presets the chromatin landscape around AR target sites for subsequent AR recruitment. Upon androgen stimulation, preoccupied TCF7L2 may mediate a tighter regulation of AR targets by imposing an additional barrier to transcriptional activation, whereas the increased recruitment of AR and gradual dissociation of TCF7L2 enable a greater activation of these AR targets during androgen exposure.

TCF7L2 is known as a downstream effector of the Wnt/ $\beta$-catenin signaling pathway and actively represses gene transcription in the absence of Wnt ligands through association with ubiquitous corepressors such as (TLE)/ Groucho family members. Interestingly, it has been suggested that FOXA1-dependent recruitment of TLE3 represses the activities of target enhancers by blocking the local recruitment of other factors for gene activation (Sekiya and Zaret 2007). Our findings suggest that the cooperation between TCF7L2 and FOXA1 may help recruit TLEs to specific target genes. TCF7L2 is a sequencespecific HMG-box-containing protein with the ability to induce DNA bending by binding to the minor groove of the DNA helix, thus mediating DNA looping for long-distance interactions of distal enhancers and proximal promoters (van Houte et al. 1993; Giese et al. 1995; Love et al. 1995). Through ChIP-seq analysis, we identified that the majority of TCF7L2-binding sites are located at distal intergenic and intronic regions, consistent with its regulatory role on distal enhancers. The genomic colocalization with FOXA1 provides TCF7L2 accessibility to AR-binding sites, given that FOXA1 resides at $90 \%$ of all of the TCF7L2-AR sites. Importantly, these AR-FOXA1-TCF7L2-overlapping sites are significantly associated with androgen-induced genes such as MYC.

MYC is an immediate early response gene downstream from many oncogenic signaling pathways and is tightly regulated by various mechanisms through many cisregulatory elements identified within its proximal promoters and distal enhancer regions (Hurley et al. 2006; Levens 2010). We demonstrate that AR and FOXA1 cooperate to modulate DHT-stimulated MYC activation, and TCF7L2 represses both basal and AR-induced MYC expression by acting on the distal enhancers upstream of the MYC gene. The presence of FOXAl at the same regions may facilitate $A R$ recruitment for immediate activation of $M Y C$ in response to androgen stimulation. Beyond the transcriptional activation of $M Y C$, the ARstimulated HER2/HER3 pathway also vigorously enhances MYC activity through destabilizing MAD1, the competitor of MYC for MAX interaction. Hence, targeting of AR and AR-regulated signaling pathway not only inhibits MYC induction but also interrupts the critical interaction between MYC and MAX. In normal cells, MYC transcriptionally activates the PTEN tumor suppressor to repress the PI3K/AKT pathway, subsequently leading to EZH2-mediated gene repression (Kaur and Cole 2013). However, the homeostatic balance that controls normal growth is disrupted in cancer cells through a variety of mechanisms. In molecular apocrine breast cancers, the amplification of HER2 coupled with AR activation dramatically enhances PI3K signaling and the oncogenic function of MYC.

Recent high-throughput analyses of MYC-binding sites and associated gene expression profiles suggested that

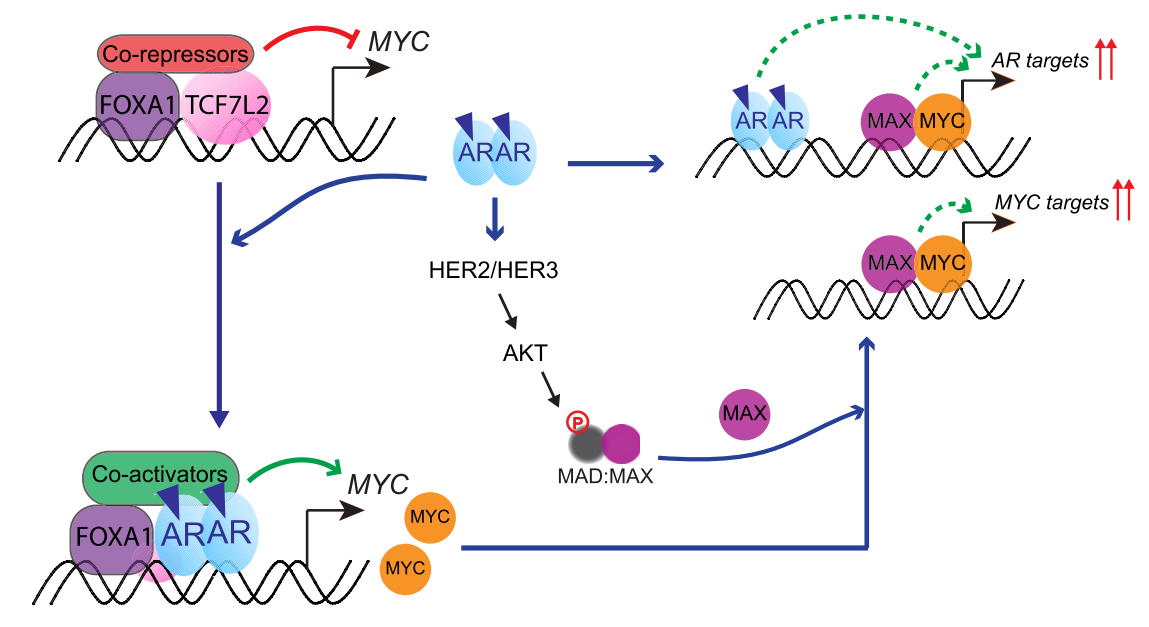

Figure 8. A model of the regulation and function of MYC in the androgen signaling pathway in molecular apocrine breast cancers. 
MYC occupancy is often insufficient to induce changes on mRNA levels of its target genes (Li et al. 2003; Zeller et al. 2006; Kim et al. 2010). In addition, studies of genetic mouse models indicated that MYC necessitates additional genetic alterations in vivo to enable its tumorigenic potential (for review, see Dang 2012). Recent studies showed that MYC serves as an amplifier of the existing active transcriptional programs rather than binding and activating a new set of genes (Lin et al. 2012; Nie et al. 2012). We observed that the MYC cistrome and MYCbound genes are very similar between MDA-MB-453 breast cancer cells, hES cells, and K562 leukemic cells. However, the functional role of MYC in different cellular contexts appears to be cell type-specific. We showed that MYC specifically enforces androgen-regulated gene activation by acting on the promoters of direct MYC targets and a subset of AR targets at which AR mainly occupies the enhancer regions. These findings suggest that AR may employ MYC as a primary response target to amplify the androgen-regulated transcriptional program in molecular apocrine breast cancer cells. Previous studies have shown that MYC binds to actively transcribed genes, and elevated MYC expression does not increase the number of activated genes (Lin et al. 2012; Nie et al. 2012). Our findings suggest that androgenmediated MYC induction specifically increases expression of a subgroup of androgen-up-regulated genes in molecular apocrine breast cancer cells, although globally MYC occupancy is observed at a larger number of gene promoters. Thus, this study provides critical insights into the cell type-specific role of MYC in regulating androgen signaling in breast cancer and also suggests that the context-specific activation of MYC and the ability of MYC to co-opt the functions of other key transcription factors enable MYC to coordinate differential gene expression programs in a cell type-dependent manner.

In summary, these findings reveal novel mechanistic details of an AR-centered regulatory network and define a positive feed-forward loop involving MYC in regulating androgen-dependent transcription in molecular apocrine breast cancers. A deeper molecular understanding of the regulation and function of MYC should facilitate development of rational, mechanism-based therapeutics to target the androgen signaling pathway in breast cancers.

\section{Materials and methods}

\section{Cell culture}

MDA-MB-453 breast cancer cells were grown as described previously (Ni et al. 2011). In all experiments, the cells were cultured in hormone-depleted medium for $2 \mathrm{~d}$, followed by exposure to vehicle control or $10 \mathrm{nM}$ DHT. Bicalutamide, lapatinib, and IWP2 were added at final concentrations of $2 \mu \mathrm{M}, 100 \mathrm{nM}$, and $1 \mu \mathrm{M}$, respectively.

\section{ChIP and ChIP-seq}

ChIP was performed as previously described (Ni et al. 2011). Library preparation for next-generation sequencing was performed according to the manufacturer's instructions (Illumina) with 10 ng of ChIP DNA. Single paired libraries were sequenced on the Illumina HiSeq2000 platform. Antibodies against TCF7L2 (sc8631, Santa Cruz), AR (sc-816, Santa Cruz), FOXA1 (ab23738, Abcam), and MYC (sc-764, Santa Cruz Biotechnology) were used in the direct ChIP and ChIP-seq assays. The primers used in direct ChIP-qPCR analysis are described in the Supplemental Material.

\section{RNAi}

Lentiviral shRNA constructs in the pLKO vector were obtained from the RNAi Core Facility of Dana-Farber Cancer Institute. The shRNA target sequences used were TCF7L2 (no. 3, 5'-CGA ACCTATCTCCAGATGAAA- ${ }^{\prime}$; no. 4, 5' - CGTCACCAAGTTTA GAATA-3'; and no. 5, 5'-GCCTCTTATCACGTACAGCAA-3'), FOXA1 (5'-GAACACCTACATGACCATGAA-3'), and AR (5'CCTGCTAATCAAGTCACACAT-3'). Lentivirus preparation and transduction of cells were carried according to the online protocol (http://www.broadinstitute.org/rnai/public/resources/ protocols). The SMARTpool siRNAs of control and MYC were from Dharmacon and were transfected into MDA-MB-453 cells using Lipofectamine RNAiMAX reagent (Invitrogen) according to the manufacturer's instructions.

\section{$R N A$ isolation and RT-PCR}

Total RNA was isolated using the RNeasy minikit (Qiagen) according to the manufacturer's protocol. cDNA was synthesized with the high-capacity cDNA reverse transcription kit (Applied Biosystems). Real-time quantitative RT-PCR was performed using the SYBR Green mix from Applied Biosystems. Primer sequences are listed in the Supplemental Material.

\section{Coimmunoprecipitation and Western blot}

The endogenous coimmunoprecipitation experiments were performed using nuclear extracts as described previously (Xu et al. 2010). Briefly, $5 \mathrm{mg}$ of nuclear extracts was incubated with $5 \mu \mathrm{g}$ of the indicated antibody on a rotator overnight at $4^{\circ} \mathrm{C}$. The protein complexes were precipitated by addition of protein $\mathrm{G} / \mathrm{A}$-agarose beads (Roche) with incubation for $6 \mathrm{~h}$ at $4^{\circ} \mathrm{C}$. The beads were washed four times for $15 \mathrm{~min}$ and then boiled for 5 min in protein sample buffer (Bio-Rad). The antibodies used for immunoprecipitation included TCF7L2 (sc-8631, Santa Cruz Biotechnology), FOXA1 (ab23738, Abcam), and MAX (no. 4732, Cell Signaling). Western blot analysis was performed as described previously (Ni et al. 2011) using antibodies against AR (sc-7305), MYC (sc-764), MAD1 (sc-222), MAX (sc-765), HER3 (sc-7390), TBP (sc-204), and GAPDH (sc-25778) from Santa Cruz Biotechnology; TCF7L2 (clone 1B1) from Sigma-Aldrich; FOXA1 (ab40868) from Abcam; and p-HER3 (no.4791), p-AKT (no.4060), and AKT (no.9272) from Cell Signaling. The antibody against p-MAD1(S145) was kindly provided by Dr. Junying Yuan (Harvard Medical School, Boston, MA).

\section{$3 C$}

3C assay was performed as described previously (Pomerantz et al. 2009; Xu et al. 2010) with some modifications. Briefly, after treatment of vehicle or DHT for $4 \mathrm{~h}$, MDA-MB-453 cells were cross-linked with $1 \%$ formaldehyde for $10 \mathrm{~min}$. The cell pellets were lysed in ice-cold lysis buffer $(10 \mathrm{mM}$ Tris- $\mathrm{HCl}$ at $\mathrm{pH} 8.0$, $10 \mathrm{mM} \mathrm{NaCl}, 0.2 \% \mathrm{NP}-40,1 \mathrm{mM}$ dithiothreitol) for $10 \mathrm{~min}$, and the cell nuclei were collected and resuspended in the restriction enzyme buffer for Hind III with incubation for $1 \mathrm{~h}$ at $37^{\circ} \mathrm{C}$. Triton 
$\mathrm{X}-100$ was added to a final concentration of $1.8 \%$ followed by overnight digestion of HindIII at $37^{\circ} \mathrm{C}$. DNA ligation was performed for $24 \mathrm{~h}$ at $16^{\circ} \mathrm{C}$. After reverse cross-linking overnight, the ligated samples were incubated with proteinase $\mathrm{K}$ for $2 \mathrm{~h}$. The DNA samples were then purified by phenol extraction and ethanol precipitation. The primers for quantitative real-time PCR are provided in the Supplemental Material.

\section{RNA-seq}

After transfection with control or MYC siRNA for 48 h, MDAMB-453 cells were exposed to $10 \mathrm{nM}$ DHT or vehicle for $6 \mathrm{~h}$ and collected for RNA-seq assays. Total RNA was isolated using RNeasy mini kit (Qiagen), and oligo d(T)25 beads (New England Biolabs) were then used to purify the polyadenylated mRNA. One nanogram of mRNA was used for library preparation using Encore Complete RNA-seq kit (NuGEN) according to the manufacturer's instructions, followed by sequencing on the Illumina HiSeq2000 platform.

\section{Bioinformatic analysis}

ChIP-seq peak calling was performed using the MACS package (Zhang et al. 2008) with a $P$-value cutoff of $1 \times 10^{-12}$. The peaks showing more than threefold enrichment were used for downstream analysis. The analysis of motif enrichment in peak regions was evaluated using the Seqpos algorithm (Liu et al. 2011). For the correlation analysis between differential gene expression and transcription factor binding, we first calculated the percentage of DHT-regulated genes and the percentage of all genes with binding sites of AR, FOXA1, or TCF7L2 in the indicated combinations at the \pm 20 -kb region from the TSS. The ratio between these two percentages was determined as fold enrichment. Fisher's exact test was used to assess the statistical significance in the correlation analysis. Differential expression analysis was performed using Cufflinks (Trapnell et al. 2010), with the FDR cutoff of 0.05 and fold change more than two. ChIP-seq and RNA-seq data have been deposited in the Gene Expression Omnibus database under GSE45203.

\section{Acknowledgments}

We thank members of the Brown laboratory for technical support and helpful discussions. This work was supported by funding from the Breast Cancer Research Foundation (to M.B.), the National Cancer Institute (P01CA080111 to M.B.), the National Human Genome Research Institute (R01HG004069 to X.S.L), and a Department of Defense Award (W81XWH-10-1-0037 to M.N.).

\section{References}

Ayer DE, Kretzner L, Eisenman RN. 1993. Mad: A heterodimeric partner for Max that antagonizes Myc transcriptional activity. Cell 72: 211-222.

Carroll JS, Liu XS, Brodsky AS, Li W, Meyer CA, Szary AJ, Eeckhoute J, Shao W, Hestermann EV, Geistlinger TR, et al. 2005. Chromosome-wide mapping of estrogen receptor binding reveals long-range regulation requiring the forkhead protein FoxA1. Cell 122: 33-43.

Chen X, Xu H, Yuan P, Fang F, Huss M, Vega VB, Wong E, Orlov YL, Zhang W, Jiang J, et al. 2008. Integration of external signaling pathways with the core transcriptional network in embryonic stem cells. Cell 133: 1106-1117.

Chou CK, Lee DF, Sun HL, Li LY, Lin CY, Huang WC, Hsu JM, Kuo HP, Yamaguchi H, Wang YN, et al. 2009. The suppression of MAD1 by AKT-mediated phosphorylation activates
MAD1 target genes transcription. Mol Carcinog 48: 10481058.

Dang CV. 2012. MYC on the path to cancer. Cell 149: 22-35.

Eeckhoute J, Lupien M, Meyer CA, Verzi MP, Shivdasani RA, Liu XS, Brown M. 2009. Cell-type selective chromatin remodeling defines the active subset of FOXA1-bound enhancers. Genome Res 19: 372-380.

Farmer P, Bonnefoi H, Becette V, Tubiana-Hulin M, Fumoleau P, Larsimont D, Macgrogan G, Bergh J, Cameron D, Goldstein D, et al. 2005. Identification of molecular apocrine breast tumours by microarray analysis. Oncogene 24: 4660-4671.

Giese K, Kingsley C, Kirshner JR, Grosschedl R. 1995. Assembly and function of a TCR $\alpha$ enhancer complex is dependent on LEF-1-induced DNA bending and multiple protein-protein interactions. Genes Dev 9: 995-1008.

Hurley LH, Von Hoff DD, Siddiqui-Jain A, Yang D. 2006. Drug targeting of the c-MYC promoter to repress gene expression via a G-quadruplex silencer element. Semin Oncol 33: 498512.

Kaur M, Cole MD. 2013. MYC acts via the PTEN tumor suppressor to elicit autoregulation and genome-wide gene repression by activation of the Ezh2 methyltransferase. Cancer Res 73: 695-705.

Kim J, Chu J, Shen X, Wang J, Orkin SH. 2008. An extended transcriptional network for pluripotency of embryonic stem cells. Cell 132: 1049-1061

Kim J, Woo AJ, Chu J, Snow JW, Fujiwara Y, Kim CG, Cantor AB, Orkin SH. 2010. A Myc network accounts for similarities between embryonic stem and cancer cell transcription programs. Cell 143: 313-324.

Levens D. 2010. You don't muck with MYC. Genes Cancer 1: 547-554.

Li Z, Van Calcar S, Qu C, Cavenee WK, Zhang MQ, Ren B. 2003. A global transcriptional regulatory role for c-Myc in Burkitt's lymphoma cells. Proc Natl Acad Sci 100: 8164-8169.

Lim E, Ni M, Hazra A, Tamimi RM, Brown M. 2012. Elucidating the role of androgen receptor in breast cancer. Clin Investig 2: 1003-1011.

Lin CY, Loven J, Rahl PB, Paranal RM, Burge CB, Bradner JE, Lee TI, Young RA. 2012. Transcriptional amplification in tumor cells with elevated c-Myc. Cell 151: 56-67.

Liu T, Ortiz JA, Taing L, Meyer CA, Lee B, Zhang Y, Shin H, Wong SS, Ma J, Lei Y, et al. 2011. Cistrome: An integrative platform for transcriptional regulation studies. Genome Biol 12: R83.

Love IJ, Li X, Case DA, Giese K, Grosschedl R, Wright PE. 1995. Structural basis for DNA bending by the architectural transcription factor LEF-1. Nature 376: 791-795.

Lupien M, Eeckhoute J, Meyer CA, Wang Q, Zhang Y, Li W, Carroll JS, Liu XS, Brown M. 2008. FoxAl translates epigenetic signatures into enhancer-driven lineage-specific transcription. Cell 132: 958-970.

Mao CD, Byers SW. 2011. Cell-context dependent TCF/LEF expression and function: Alternative tales of repression, derepression and activation potentials. Crit Rev Eukaryot Gene Expr 21: 207-236.

Ni M, Chen Y, Lim E, Wimberly H, Bailey ST, Imai Y, Rimm DL, Liu XS, Brown M. 2011. Targeting androgen receptor in estrogen receptor-negative breast cancer. Cancer Cell 20: 119-131.

Nie Z, Hu G, Wei G, Cui K, Yamane A, Resch W, Wang R, Green DR, Tessarollo L, Casellas R, et al. 2012. c-Myc is a universal amplifier of expressed genes in lymphocytes and embryonic stem cells. Cell 151: 68-79.

Pomerantz MM, Ahmadiyeh N, Jia L, Herman P, Verzi MP, Doddapaneni H, Beckwith CA, Chan JA, Hills A, Davis M, 
Ni et al.

et al. 2009. The $8 \mathrm{q} 24$ cancer risk variant rs6983267 shows long-range interaction with MYC in colorectal cancer. Nat Genet 41: 882-884.

Rhodes DR, Kalyana-Sundaram S, Tomlins SA, Mahavisno V, Kasper N, Varambally R, Barrette TR, Ghosh D, Varambally S, Chinnaiyan AM. 2007. Molecular concepts analysis links tumors, pathways, mechanisms, and drugs. Neoplasia 9: $443-454$.

Robinson JL, Macarthur S, Ross-Innes CS, Tilley WD, Neal DE, Mills IG, Carroll JS. 2011. Androgen receptor driven transcription in molecular apocrine breast cancer is mediated by FoxA1. EMBO J 30: 3019-3027.

Sekiya T, Zaret KS. 2007. Repression by Groucho/TLE/Grg proteins: Genomic site recruitment generates compacted chromatin in vitro and impairs activator binding in vivo. Mol Cell 28: 291-303.

Serandour AA, Avner S, Percevault F, Demay F, Bizot M, Lucchetti-Miganeh C, Barloy-Hubler F, Brown M, Lupien M, Metivier R, et al. 2011. Epigenetic switch involved in activation of pioneer factor FOXA1-dependent enhancers. Genome Res 21: 555-565.

Shin H, Liu T, Manrai AK, Liu XS. 2009. CEAS: Cis-regulatory element annotation system. Bioinformatics 25: 2605-2606.

Tan SK, Lin ZH, Chang CW, Varang V, Chng KR, Pan YF, Yong EL, Sung WK, Cheung E. 2011. AP-2 $\gamma$ regulates oestrogen receptor-mediated long-range chromatin interaction and gene transcription. EMBO J 30: 2569-2581.

Trapnell C, Williams BA, Pertea G, Mortazavi A, Kwan G, van Baren MJ, Salzberg SL, Wold BJ, Pachter L. 2010. Transcript assembly and quantification by RNA-Seq reveals unannotated transcripts and isoform switching during cell differentiation. Nat Biotechnol 28: 511-515.

van Houte L, van Oers A, van de Wetering M, Dooijes D, Kaptein R, Clevers H. 1993. The sequence-specific high mobility group 1 box of TCF-1 adopts a predominantly $\alpha$-helical conformation in solution. J Biol Chem 268: 1808318087.

Wolfer A, Wittner BS, Irimia D, Flavin RJ, Lupien M, Gunawardane RN, Meyer CA, Lightcap ES, Tamayo P, Mesirov JP, et al. 2010. MYC regulation of a 'poor-prognosis' metastatic cancer cell state. Proc Natl Acad Sci 107: 3698-3703.

Woodfield GW, Horan AD, Chen Y, Weigel RJ. 2007. TFAP2C controls hormone response in breast cancer cells through multiple pathways of estrogen signaling. Cancer Res 67: 8439-8443.

Xu J, Sankaran VG, Ni M, Menne TF, Puram RV, Kim W, Orkin SH. 2010. Transcriptional silencing of $\gamma$-globin by BCL11A involves long-range interactions and cooperation with SOX6. Genes Dev 24: 783-798.

Zeller KI, Zhao X, Lee CW, Chiu KP, Yao F, Yustein JT, Ooi HS, Orlov YL, Shahab A, Yong HC, et al. 2006. Global mapping of c-Myc binding sites and target gene networks in human B cells. Proc Natl Acad Sci 103: 17834-17839.

Zhang Y, Liu T, Meyer CA, Eeckhoute J, Johnson DS, Bernstein BE, Nussbaum C, Myers RM, Brown M, Li W, et al. 2008. Model-based analysis of ChIP-Seq (MACS). Genome Biol 9: R137.

Zhu J, Blenis J, Yuan J. 2008. Activation of PI3K/Akt and MAPK pathways regulates Myc-mediated transcription by phosphorylating and promoting the degradation of Mad1. Proc Natl Acad Sci 105: 6584-6589. 


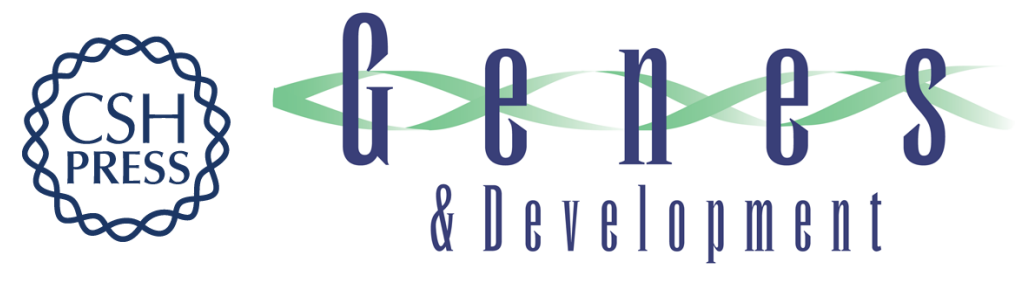

\section{Amplitude modulation of androgen signaling by c-MYC}

Min Ni, Yiwen Chen, Teng Fei, et al.

Genes Dev. 2013, 27: originally published online March 25, 2013

Access the most recent version at doi:10.1101/gad.209569.112

Supplemental

Material

References

License

Email Alerting Service
http://genesdev.cshlp.org/content/suppl/2013/03/20/gad.209569.112.DC1

This article cites 37 articles, 11 of which can be accessed free at: http://genesdev.cshlp.org/content/27/7/734.full.html\#ref-list-1

Receive free email alerts when new articles cite this article - sign up in the box at the top right corner of the article or click here.

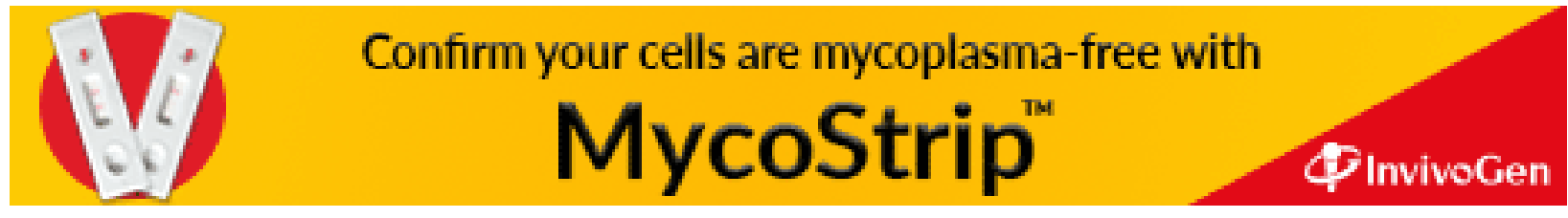

